\title{
A hybrid particle-number and particle model for efficient solution of population balance equations
}

\author{
Astrid Boje $^{\mathrm{a}}$, Jethro Akroyd ${ }^{\mathrm{a}}$, Markus Kraft $\mathrm{At}^{\mathrm{a}, \mathrm{b}, \mathrm{c}, *}$ \\ ${ }^{a}$ Department of Chemical Engineering and Biotechnology, University of Cambridge, West \\ Cambridge Site, Philippa Fawcett Drive, Cambridge, CB3 0AS, United Kingdom \\ ${ }^{b}$ Cambridge Centre for Advanced Research and Education in Singapore (CARES), \\ CREATE Tower, 1 CREATE Way, 138602, Singapore \\ ${ }^{c}$ School of Chemical and Biomedical Engineering, Nanyang Technological University, 62 \\ Nanyang Drive, Singapore, 637459
}

\section{Abstract}

This work presents a hybrid particle-number and particle model to improve efficiency in solving population balance equations for type spaces spanning spherical and aggregate particles. The particle-number model tracks simpler, spherical particles cheaply by storing only the number of particles with a given one-dimensional internal coordinate, while the particle model allows resolution of the detailed aggregate structure that occurs due to collision and coagulation between particles by storing distinct computational entries for each particle. This approach is exact if primary particles are defined by their monomer count and the particle-number model increments in single monomers. A stochastic method is used to solve the population balance equations for the combined type space. The hybrid method works well for large ensembles ( $>2^{12}$ particles) with a detailed particle model, where per-

\footnotetext{
* Markus Kraft

Email address: \{mk306@cam.ac.uk\} (Markus Kraft)

$U R L$ : https://como.cheng.cam.ac.uk/ (Markus Kraft)
} 
forming a finite number of particle-number updates is demonstrated to be 40-50\% cheaper than updating an equivalent ensemble of discrete particles. These savings can be traded for a larger sample volume to increase the resolution in the particle size distribution or more repeat runs to reduce the total error. Run time improvements are curtailed at very high surface growth and coagulation rates due to the fixed cost of growth updates on the large aggregates formed; however, the hybrid method is still attractive in this case as its primary purpose is to reduce error by preventing saturation of the ensemble with simple particles at high inception rates.

Keywords: hybrid method, particle model, particle-number model, high rate, particle processes, population balance

1. Introduction

The dynamics of particle formation and growth are of interest across 3 a wide range of systems from flame synthesis of nanoparticles [1, 2] and 4 crystallisation [3] to large scale systems such as atmospheric [4, 5] and as5 trophysical $[6,7]$ studies. The evolution of a particle system through time integro-differential equation which describes changes in the internal coordinates of the particles (e.g. mass, surface area, chemical composition and structure) due to processes such as inception, collision, surface reaction or condensation, and fragmentation. The complexity of real systems precludes analytical solutions; thus numerical methods have been developed. Numerical solutions require a model for the particle type space and a method for solving the PBE. 
The particle type space is typically high dimensional, with each particle described by up to thousands of internal coordinates which correspond to the diversity of morphologies and surface chemistries that can be formed [8]. The simplest type space model is a spherical particle model, which represents particles as spheres of constant composition and density; thus only a one dimensional type space is required. This assumes that lasting collision (i.e. coagulation) events are followed by instantaneous coalescence to a larger spherical particle [9]. More detail is incorporated into surface area and volume models [10], where these properties are added for coagulating particles. This allows more structural information to be tracked; however, these models require adaptations to deal with processes such as surface reaction and sintering (e.g. a fractal dimension is assumed).

The most detailed particle models are primary particle models. These resolve the connectivity of "primary particles" (particles formed by inception) following coagulation events and describe particle structure e.g. shared surface area and centre-to-centre distance between particles [11]. Detailed particle models have been used to study synthesis of soot [12, 13, 14], $\mathrm{SiO}_{2}$ $[15,16]$, silicon [17] and $\mathrm{TiO}_{2}[18,19,11]$. Detailed particle models have been shown to provide important additional information when the particle system is polydisperse or the coagulation and sintering timescales are similar [20].

The numerical solution of the PBE becomes more challenging with increasing type space complexity. Low dimensional type spaces allow direct integration of the ordinary differential equations (ODE) through transport of the moments of the particle size distribution (PSD) or discretization. Stadnichuk et al. [21] and Smith et al. [22] describe iterative schemes for efficient 
steady state solutions and $\mathcal{H}$-matrices are used as low rank, separable approximations to the coagulation and fragmentation kernels in Koch et al. [23] to reduce computational cost and memory requirements.

The method of moments (MOM) approach solves finitely many moments of the particle size distribution by multiplying the PBE by $k^{\text {th }}$ powers of a property and integrating over the type space. This approach is computationally efficient, although closure problems exist for coagulation kernels involving fractional or negative moments and processes requiring the pointwise particle concentrations (shrinkage). Closure issues are treated by interpolation e.g. MOMIC [24, 25, 26, 27] or quadrature e.g. QMOM [28, 29], DQMOM [30,31]. The moment projection method has been proposed to handle shrinkage problems [32].

Sectional methods are a popular choice of ODE-based method. These discretize the PSD into sections/bins within which the PSD is modelled either with step functions or polynomials. A number of adaptations have been proposed to e.g. conserve mass and particle number [33], handle discontinuities in the number distribution and numerical diffusion due to surface reaction $[34,35,36]$, and treat sintering [37]. However, sectional methods must approximate properties of the PSD within the discretized sections, are expensive compared with MOM, and higher order variants can suffer from stability issues [8].

Discretization-based solvers applying finite difference [38], finite volume [39] and finite element [40] methods are widely used for low dimensional type spaces. Matveev et al. [38] propose low rank skeleton approximations for the kernel matrix to exploit fast convolutions and reduce complexity. Such 
techniques can accommodate multidimensional problems with several internal coordinates in the particle model (e.g. 2-5 coordinates in Matveev et al. [41]). These methods become prohibitively expensive for higher dimensional type spaces for example, the thousands of dimensions required to describe aggregate particle structure including all possible configurations and sizes of the constituent primary particles.

Stochastic (Monte Carlo) methods solve the PBE by performing events probabilistically on a finite ensemble of computational particles which can have arbitrarily many internal coordinates. Monte Carlo methods are currently the only viable method for using very high dimensional particle type spaces. The accuracy of these methods is controlled by the number of computational particles used and the number of repeat runs with different random seeds. This can be computationally taxing under high rate conditions, such as those used in our recent study of industrial $\mathrm{TiO}_{2}$ synthesis [19] because a large particle ensemble is required to resolve the polydisperse PSD and the surface structure of the particles evolves rapidly. In Monte Carlo methods, convergence to the exact solution is expected with increasing sample size. This can be demonstrated numerically [16, 42], and has been shown theoretically in several studies $[43,44,45]$.

In previous work, the stochastic approach has been refined with several techniques to reduce variance e.g. doubling [46] and mass flow algorithms [43] and weighted particle methods [47, 48, 49], and improve efficiency e.g. fictitious jumps and majorant kernels [50], linear process deferment algorithm [51]. A split solution method has been proposed for studying gelation processes, to reduce the chance of stochastic effects forming metastable states 
[52]: the ODEs for particles smaller than size $N_{1}$ are treated deterministically, those for particles of sizes between $N_{1}$ and $N_{2}$ are treated stochastically, and larger particles are removed (the gelled mass).

The purpose of this paper is to introduce a hybrid particle-number/particle (PN/P) model to handle broad particle size distributions where aggregate morphology is important. In the case of high particle inception rates, it becomes computationally challenging to resolve the less abundant, larger particle aggregates, especially when particle surface processes such as heterogeneous reaction are also significant. The proposed PN/P model exploits the simpler morphology of particles in some regions of the type space; small particles are treated using a particle-number method, while large particles and aggregates are resolved with a detailed type space model. If the detailed model employs a one dimensional description of primary particles, the $\mathrm{PN} / \mathrm{P}$ approach is exact.The algorithm presented here adapts the standard direct simulation algorithm (DSA), including majorant techniques and LPDA. The extension to weighted particle methods could be considered in future work.

This paper is structured as follows: The PBE is stated in Section 2. Two particle systems are defined using particle-number and detailed particle models in Section 3. The processes that transfer mass between the particle systems are then described in general terms. The stochastic method used is outlined in Section 4. Section 5 presents numerical studies of the convergence and performance of the hybrid model compared to a single particle model. Various configurations of a simplified $\mathrm{TiO}_{2}$ test are used and the relevant rate forms are provided explicitly. 


\section{Population balance equation}

The concentration of particles of a given multivariate type $x \in \mathcal{E}$, where $\mathcal{E}$ is called the type space and describes all possible particles, can be evolved by the Smoluchowski coagulation equation [47], extended to include inception, surface changes and flow. Here, we consider flow in an ideal, constant volume, continuously stirred tank reactor (CSTR) (Eq. (1)).

$$
\begin{aligned}
\frac{\mathrm{d} n(t, x)}{\mathrm{d} t}= & I(x)+\frac{1}{2} \sum_{\substack{y, z \in \mathcal{E}: \\
y+z=x}} K(y, z) n(t, y) n(t, z) \\
& -\sum_{y \in \mathcal{E}} K(x, y) n(t, x) n(t, y) \\
& +\sum_{\substack{y \in \mathcal{E}: \\
g_{\mathrm{SG}}(y)=x}} \beta_{\mathrm{SG}}(y) n(t, y)-\beta_{\mathrm{SG}}(x) n(t, x) \\
& +\frac{1}{\tau_{\mathrm{CSTR}}} \sum_{j=1}^{N_{\mathrm{in}}} f^{[j]}\left(n_{\mathrm{in}}^{[j]}(t, x)-n(t, x)\right)
\end{aligned}
$$

9 $\mathrm{r}$ of $\mathrm{p}$ s is is the residence time in the CSTR. In the case of $N_{\text {in }}$ inflow streams, $f^{[j]}$ is the volumetric feed fraction of the $j^{\text {th }}$ stream. 




Figure 1: Mass transfer from the gas phase to the particle systems by inception and surface reaction, and mass transfer from the particle-number model to the particle model by coagulation and surface growth beyond the threshold size $\left(N_{\text {thresh }}\right)$.

\section{Particle systems}

Monte Carlo methods employ a finite ensemble of computational particles to model the diverse assortment of particles in the physical system. A computational particle $P_{i}$ has a distinct, possibly multivariate type, $x_{i}$.

In this work, a hybrid particle-number/particle model is proposed wherein the particle type space is split such that $\mathcal{E}=(\mathcal{M} \cup \mathcal{X})$. This allows different levels of detail to be used to describe particles in the spaces $\mathcal{M}$ and $\mathcal{X}$ (Fig. 1).

\subsection{Space of small, spherical particles, $\mathcal{M}$}

Let the particle type space consisting of small, spherical particles (primary particles) be defined as $\mathcal{M}$. Particles in this space have a single internal coordinate for number of monomers, with different sizes $i \in\left[1, N_{\text {thresh }}\right]$ where $i=1$ is a single molecular unit and $N_{\text {thresh }}$ is the size of the largest particle that is tracked by the particle-number model before transfer to the space of 
aggregate particles, $\mathcal{X}$. The particle-number $(\mathrm{PN})$ system is written:

$$
z_{\mathcal{M}}(t)=\left(x_{1}, \ldots, x_{N_{\text {thresh }}}\right)
$$

$140 \quad$ where

$$
x_{i}(t) \in \mathcal{M}, \quad i=1, \ldots, N_{\text {thresh }}, \quad t \geq 0
$$

${ }_{141}$ and $N_{i}=N\left(x_{i}\right)$ is the number of particles that have type $x_{i}$. For contin142 143 uous functions $\phi$, the following convergence property can be maintained as the sample volume, $V_{\mathrm{smp}}$, increases:

$$
\int_{\mathcal{M}} \phi(x) n(t, d x)=\lim _{V_{\mathrm{smp}} \rightarrow \infty} \frac{1}{V_{\mathrm{smp}}} \sum_{i=1}^{N_{\text {thresh }}} N_{i} \phi\left(x_{i}(t)\right) .
$$

144 Here, we use the concentration measure $n(t, d x)$ in place of the density 145

$n(t, x)$ to allow for particle type spaces with continuous and discrete components [47]. The concentration of particles with type $x_{i} \in \mathcal{M}$ is $N_{i} \cdot V_{\mathrm{smp}}^{-1}$. The type space $\mathcal{M}$ can be represented efficiently as it requires only a vector in $\mathbb{R}^{N_{\text {thresh }}}$ to produce the PSD from the number of particles in each size class.

\subsection{Space of large particles and aggregates, $\mathcal{X}$}

Let $\mathcal{X}$ be the type space for spherical particles containing more than $N_{\text {thresh }}$ monomers and all aggregate particles containing more than one primary particle. Particles in $\mathcal{X}$ need to be defined by both morphology and 
where t):

$$
P_{i}=\left(p_{1}, \ldots, p_{n_{i}}, \mathbf{C}\right) .
$$

In this work, the data structure of each particle stores a connectivity matrix $\mathbf{C}$ to track adjacent primary particles and their shared surface area (Figs. 2(b) and 2(c)). The particle model has been comprehensively described by Sander et al. [15] and Shekar et al. [16]. The shared surface area $C_{a, b}$ must be updated if connected primary particles $p_{a}, p_{b}$ undergo surface processes. Sintering is not considered in the studies presented here. Sander et al. [15] and Lindberg et al. [11] describe treatment of sintering for the current type space, assuming grain boundary diffusion to define the characteristic sintering time. It would be simple to extend this detailed particle model to track the relative positions of primary particles in each aggregate in order to resolve collisions and surface changes in more detail, as presented by our co-workers in Lindberg et al. [53].

The particle system is comprised of $N(t) \leq N_{\max }$ such particles (at time

$$
z_{\mathcal{X}}(t)=\left(x_{1}, \ldots, x_{N(t)}\right)
$$




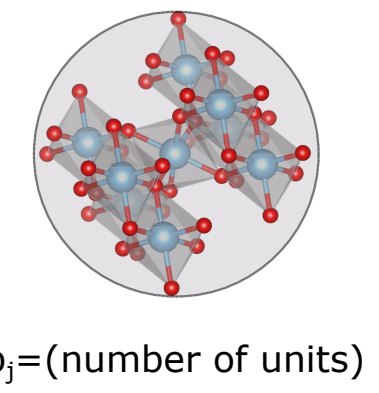

(a) Primary particle model

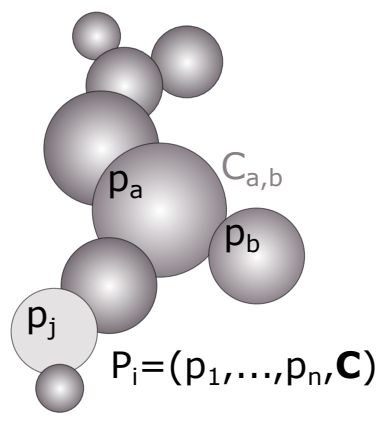

(b) Particle model



(c) Primary connectivity

Figure 2: Detailed particle type space showing a $\mathrm{TiO}_{2}$ primary particle $p_{j}$, primary particle connectivity for aggregate particle $P_{i}$ and shared surface area $C_{a, b}$ between primaries $p_{a}$ and $p_{b}$ connected by neck of radius $r_{a, b}$.

$$
x_{i}(t) \in \mathcal{X}, \quad i=1, \ldots, N(t), \quad t \geq 0
$$

$$
\int_{\mathcal{X}} \phi(x) n(t, d x)=\lim _{V_{\mathrm{smp}} \rightarrow \infty} \frac{1}{V_{\mathrm{smp}}} \sum_{i=1}^{N(t)} \phi\left(x_{i}(t)\right) .
$$

The description of multivariate particle types $x_{i}$ requires much more information for each particle; thus, a more sophisticated data structure is required to store each distinct particle separately.

\subsection{Mass transfer between the particle systems}

Eq. (1) describes the change in the PSD with time. In this work, the PSD spans two type spaces; thus, it is necessary to define how the particle 
processes affect both particle systems $z_{\mathcal{M}}(t), z_{\mathcal{X}}(t)$.

Interaction with a gas phase system

The systems of interest in this work (i.e. flame synthesis) typically involve a gas phase precursor as well as several intermediate species, and formation and reaction processes in the gas phase must be described by a chemical mechanism. Particle synthesis follows from collision between gas phase species that results in a stable configuration of molecular units (inception). Particle growth also occurs due to the reaction of gas phase species on the particle surface (surface growth) and this creates a polydisperse primary particle size distribution.

\section{Inception}

Particle inception from the gas phase intermediates occurs at a rate, $I$, that depends on the gas phase concentrations and the temperature. The inception process only acts on the space of spherical primaries, $\mathcal{M}$, and not on the space of large particles, $\mathcal{X}$. In this work, we assume that a dimer unit is the only incepting size; however, the description is transferable to any monomer index corresponding to a stable particle composition. Primary particles of type $x_{i} \in \mathcal{M}$ are created and this is modelled by incrementing the count at index $i$ in the particle-number model (Fig. 3).

\section{Surface growth}

All particles in the two type spaces can experience surface growth, at a rate, $\beta_{\mathrm{SG}}$, that is dependent on the gas phase reactant concentrations and temperature, and the particle surface area. Surface growth results in a change 


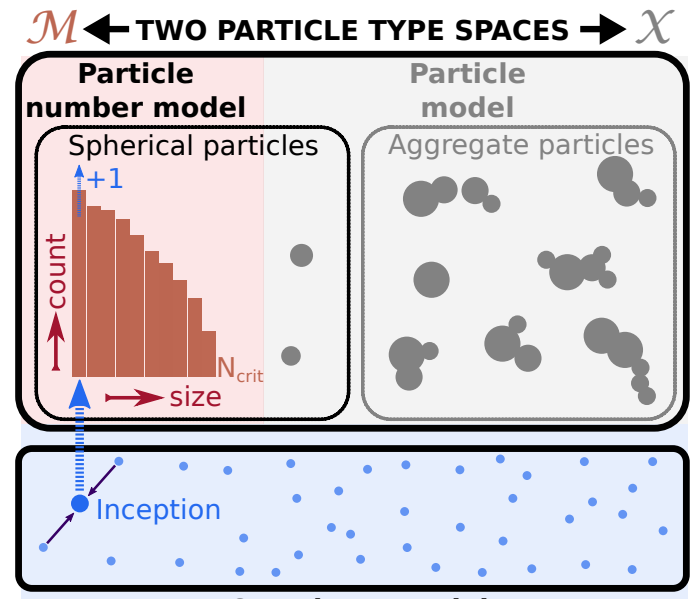

Gas phase model

Figure 3: Interaction between the gas phase and the particle-number system by inception of primary particles following gas phase collisions.

in particle type according to the surface growth function, $g_{\mathrm{SG}}$, with the following effects:

1. A particle described by the particle-number model with type $x_{i} \in \mathcal{M}$ is transformed to type $x_{j}=g_{\mathrm{SG}}\left(x_{i}\right), i<j$. If the new size is still in $\mathcal{M}$, i.e. $j \leq N_{\text {thresh }}$, the indices $i$ and $j$ are altered accordingly (Fig. 4, solid horizontal arrows).

2. If the new size exceeds the threshold size, i.e. $j>N_{\text {thresh }}$, the particle is transferred to the detailed particle model, by creation of a new particle consisting of a single primary, with type $x_{j} \in \mathcal{X}$ (Fig. 4, curved horizontal arrow).

3. Particles of type $x \in \mathcal{X}$, are transformed to larger type $y=g_{\mathrm{SG}}(x)$, $y \in \mathcal{X}$ (Fig. 4, dashed arrows). 


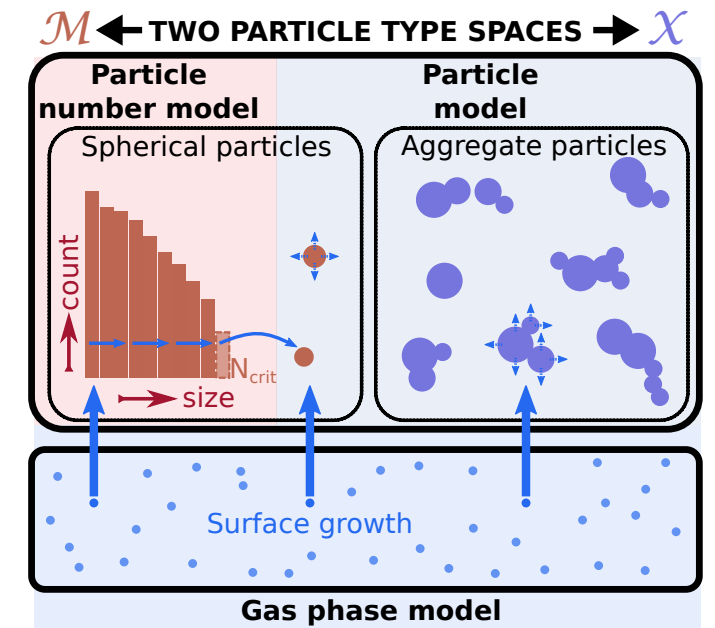

Figure 4: Interaction between the gas phase and both particle systems by surface reaction (surface reaction beyond the threshold size $N_{\text {thresh }}$ in the particle-number model causes transfer of particles to the particle model).

\section{Coagulation}

Coagulation events can occur between any two particles across both type spaces $(\mathcal{M} \cup \mathcal{X})$. This transfers particles from the particle-number model $($ space $\mathcal{M})$ to the detailed particle model (space $\mathcal{X}$ ) (Fig. 5). Coagulation between two particle-number model particles forms a new aggregate in the particle model (this process acts as a source term for the particle model) and reduces the number of particle-number particles by two. Coagulation between two particle model particles reduces by one the number of particles in the particle model system. Coagulation between one particle from each space reduces the number of particles in the particle-number model by one. The PN particle can be attached to the coagulating particle model particle, conserving the count in the particle model.

The coagulation operator $\mathcal{K}$ acts on $(\mathcal{M} \cup \mathcal{X})^{2}$ and produces particles in $\mathcal{X}$. The symmetric coagulation kernel for each particle pair is $K(x, y)$ where 
$x, y \in(\mathcal{M} \cup \mathcal{X})$. The rate $K(x, y)$ is defined by the type of coagulation process considered. The constant rate kernel and transition regime kernel used in this work are presented in more detail alongside the relevant numerical study. Because the primary particle model in $\mathcal{X}$ is one dimensional, there is no difference between the description of single primary particles in $\mathcal{M}$ and $\mathcal{X}$. Thus, the rate is derived in the same manner for particles in either space.

The total rate, $R_{\text {coag }}$, is:

$$
\begin{aligned}
R_{\mathrm{coag}}= & \frac{1}{2} \iint_{(\mathcal{M} \cup \mathcal{X})^{2}} K(x, y) n(d x) n(d y) \\
= & \frac{1}{2}\left[\int_{\mathcal{X}} \int_{\mathcal{X}} K(x, y) n(d x) n(d y)+\int_{\mathcal{M}} \int_{\mathcal{M}} K(x, y) n(d x) n(d y)\right] \\
& +\left[\int_{\mathcal{X}} \int_{\mathcal{M}} K(x, y) n(d x) n(d y)\right]
\end{aligned}
$$

235 For the discrete particle systems:

$$
\begin{gathered}
x_{i} \in z_{\mathcal{X}}(t), i=1, \ldots, N(t) \\
y_{i} \in z_{\mathcal{M}}(t), i=1, \ldots, N_{\text {thresh }}
\end{gathered}
$$

36 the rate can be written: 


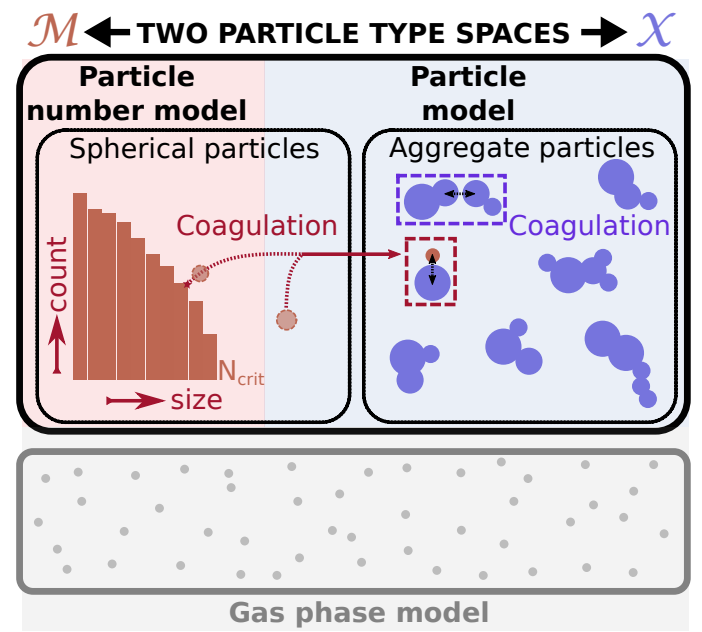

Figure 5: Interaction between the particle systems by coagulation.

$$
\begin{aligned}
R_{\text {coag }}= & \frac{1}{2 V_{\text {smp }}}\left[\sum_{i=1}^{N(t)} \sum_{\substack{j=1 \\
j \neq i}}^{N(t)} K\left(x_{i}, x_{j}\right)+\sum_{i=1}^{N_{\text {thresh }}} \sum_{\substack{j=1: \\
j \neq i}}^{N_{\text {thresh }}} K\left(y_{i}\right)<2\right. \\
& +\frac{1}{V_{\text {smp }}} \sum_{i=1}^{N(t)} \sum_{j=1}^{N_{\text {thresh }}} K\left(x_{i}, y_{j}\right) N\left(y_{j}\right) .
\end{aligned}
$$

The requirement $j \neq i \Longleftrightarrow N\left(y_{i}\right)<2$ in Eq. (3) excludes selfcoagulation from the particle-number list if there is only one particle of a given size.

Inflow

In a CSTR with particles in the inflow streams, particle inflow occurs with rate $\tau_{\text {CSTR }}^{-1}$ and particles can be added to both spaces with the following effects: 
1. If $x_{\mathrm{in}}=x_{i} \in \mathcal{M}$, the number of particles at the $i^{\text {th }}$ index of the particlenumber model is incremented: $N_{i} \leftarrow N_{i}+1, i \in\left[1, N_{\text {thresh }}\right]$.

2. If $x_{\text {in }} \in \mathcal{X}$, a new particle with type $x_{\text {in }}$ is added to the detailed particle system i.e. $z_{\mathcal{X}}(t) \leftarrow\left\{z_{\mathcal{X}}(t), P\left(x_{\text {in }}\right)\right\}$.

\section{Outflow}

In a CSTR, particle outflow occurs with rate $\tau_{\mathrm{CSTR}}^{-1}$ and particles can be removed from either particle system.

1. If $x_{\text {out }}=x_{i} \in \mathcal{M}$, the number of particles at the $i^{\text {th }}$ index of the particle-number model is decremented: $N_{i} \leftarrow N_{i}-1, i \in\left[1, N_{\text {thresh }}\right]$.

2. If $x_{\text {out }} \in \mathcal{X}$, the particle $P\left(x_{\text {out }}\right)$ is removed from the detailed particle system i.e. $z_{\mathcal{X}}(t) \leftarrow\left\{z_{\mathcal{X}}(t) \backslash P\left(x_{\text {out }}\right)\right\}$.

\section{Stochastic numerical method}

Strang operator splitting is used to couple the solution of the gas phase chemistry using an ODE solver and the solution of the particle population balance equations using a stochastic method in which the different events are performed probabilistically. This approach has been described elsewhere $[54,16]$ but is adapted here to handle the interaction between the two type space models (Algorithm B.1).

In $\mathcal{M}$, the properties (mass, diameter etc.) corresponding to each size index in the particle-number space are stored at the simulation outset and just the total particle numbers at each index i.e. 


$$
N_{i}, i=1, \ldots, N_{\text {thresh }}
$$
278

and the property sums i.e.

$$
\xi\left(z_{\mathcal{M}}\right)=\sum_{i=1}^{N_{\text {thresh }}} N_{i} \xi_{i}
$$

are updated at runtime.

The gas phase chemistry is first updated for half a time step, after which a direct simulation algorithm (DSA) is used to advance the particle population balance equations for a full time step, over a number of smaller splitting steps. Each splitting step involves repeatedly sampling a waiting time from an exponential distribution defined by the total process rate, choosing an inception or coagulation event according to their relative rates and updating the relevant particle system to reflect this event (Algorithm B.2).

If the selected process is inception, the particle-number model is adjusted by incrementing the count of particles at the index corresponding to the number of monomers in the incepting particle i.e.

$$
N_{1} \leftarrow N_{1}+1,
$$

277 and the cached property sums for the particle-number system are updated i.e. 


$$
\xi\left(z_{\mathcal{M}}(t)\right) \leftarrow \xi\left(z_{\mathcal{M}}(t)\right)+\xi_{1} .
$$

279

If the selected process is coagulation, a particle pair $\left(P_{i}, P_{j}\right)$ is selected using kernel-specific selection criteria. Majorant kernels are used in this work to simplify computation of the total coagulation rate. Fictitious jumps are used to recover the correct distribution of coagulation events, i.e. particles selected for coagulation are only updated with probability:

$$
\mathbb{P}_{i, j}=K\left(P_{i}, P_{j}\right) \cdot \hat{K}\left(P_{i}, P_{j}\right)^{-1}
$$

284 If a particle is selected from the particle-number class $\left(P_{i} \in \mathcal{M}\right)$, the index corresponding to its monomer count is decremented i.e.

$$
N_{i} \leftarrow N_{i}-1
$$

and the cached property sums are updated i.e.

$$
\xi\left(z_{\mathcal{M}}(t)\right) \leftarrow \xi\left(z_{\mathcal{M}}(t)\right)-\xi_{i}
$$

87 A new particle is created by cloning the $i^{\text {th }}$ particle from the pre-initialised particle-number list. If both particles are selected from the particle-number system, the first is added to the ensemble at this stage: 


$$
z_{\mathcal{X}}(t) \leftarrow\left\{z_{\mathcal{X}}(t), P_{i}\right\}
$$

and the second coagulates with it. Coagulation events join the colliding particles, combining their list of primaries and creating one new connection point [15].

The surface growth and sintering of adjacent primary particles is performed using a linear process deferment algorithm (LPDA). This is also a form of operator splitting which defers the particle processes that occur independently for each particle and performs them either at the end of a splitting step $t_{\text {split }}$, or during the step if the particle is selected for coagulation. This algorithm was introduced by Patterson et al. [51] to improve computational efficiency by reducing the number of times per step the algorithm halts to perform stochastic events. The splitting step is chosen to control the number of deferred particle surface updates that occur relative to the stochastic inception and coagulation events. Suitable step sizes and more details are given in the original paper [51].

The particle-number counts are updated for surface growth in a second LPDA-type sub-scheme (Algorithm B.4). This loops over all particle indices and computes the surface area dependent growth rate, samples the number of monomers to add from a Poisson distribution using this rate parameter, and uses this to determine a new index, which is incremented accordingly. 


$$
\begin{gathered}
n_{\text {add,index }} \sim \operatorname{Poi}\left(\beta_{\mathrm{SG}}\left(A_{\text {index }}\right)\right) \\
\text { newIndex }
\end{gathered}
$$

If the new index is larger than the threshold size, a new particle is created by cloning the template particle, $P_{\text {thresh }}^{\mathrm{tmp}}$, which is a primary particle of size $N_{\text {thresh }}$ monomers, from the pre-initialised particle-number list and adding (newIndex $-N_{\text {thresh }}$ ) monomers, and transferred to the detailed particle system.

Particle inflow, and outflow are performed after each splitting step. The number of particles expected to enter or leave the system over this time is sampled from a Poisson distribution with rate parameter $1 / \tau_{\mathrm{CSTR}}$. Particles are added by uniform selection from the list of particles in the inflow stream(s) followed by increasing the particle-number count $\left(x_{\text {in }} \in \mathcal{M}\right)$ or adding a particle to the ensemble $\left(x_{\mathrm{in}} \in \mathcal{X}\right)$. For each chosen particle $x_{\mathrm{in}}$, on average $V_{\mathrm{smp}} / V_{\mathrm{smp}}^{\text {in }}$ copies are added. Particles are removed by uniform selection followed by decreasing the particle-number count $\left(x_{\text {out }} \in \mathcal{M}\right)$ or deletion $\left(x_{\text {out }} \in \mathcal{X}\right)$.

\subsection{Selecting particles according to their properties}

Two particle selection processes are of interest. Uniform selection is used to choose particles to remove in outflow events, and a pair of particles to collide with a constant coagulation kernel. For more realistic coagulation kernels, selection of a pair of particles might depend on properties of the respective particles for example in the majorant proposed for the transition 
regime coagulation kernel (Table A.4), coagulation between small particles and large particles is often favoured. The selection algorithm is outlined in more detail in Algorithm B.5.

\section{Random uniform selection}

For the particle-number model with $x_{i} \in \mathcal{M}$, the index $i$ of the selected particle is selected such that:

$$
\mathbb{P}(\text { index }=i)=\frac{N_{i}}{\sum_{i=1}^{N_{\text {thresh }}} N_{i}} \quad \forall i \in\left\{1, \ldots, N_{\text {thresh }}\right\} .
$$

For the detailed particle model with $x_{i} \in \mathcal{X}$, particles $P\left(x_{i}\right)$ are selected such that:

$$
\mathbb{P}\left(P_{i}\right)=\frac{1}{N(t)} \quad \forall i \in\{1, \ldots, N(t)\}
$$

\section{Selection according to particle properties}

Let $\xi$ be a property of the particles that is defined for both type spaces e.g. mass or diameter. For the particle-number model with $x_{i} \in \mathcal{M}$, the index $i$ of the selected particle is determined using the property $\xi$ as a weighting such that:

$$
\mathbb{P}(\text { index }=i)=\frac{N_{i} \xi_{i}}{\sum_{j=1}^{N_{\text {thresh }}} N_{j} \xi_{j}} \quad \forall i \in\left\{1, \ldots, N_{\text {thresh }}\right\} \text {. }
$$


For the detailed particle model with $x_{i} \in \mathcal{X}$, particles $P\left(x_{i}\right)$ are selected using the property $\xi$ as a weighting such that:

$$
\mathbb{P}\left(P_{i}\right)=\frac{\xi\left(P_{i}\right)}{\sum_{j=1}^{N(t)} \xi\left(P_{j}\right)} \quad \forall i \in\{1, \ldots, N(t)\}
$$

\section{Numerical studies}

\subsection{Comparison with single particle type space model}

The performance of the hybrid approach is compared with a single particle type space model in which the discrete ensemble describes the full type space, and primary particles are represented by stochastic entities in the ensemble alongside aggregate particles. The latter has been the standard approach for detailed population balance models to date and is well documented in the existing literature $[19,55,17]$. Because the detailed particle model describes primary particles as spheres, the two approaches are expected to be equivalent for the same particle processes. This gives a means to validate the algorithm for the hybrid approach against the DSA. The DSA has already been compared to deterministic methods in the literature for example Maisels et al. [46], Menz et al. [42]; thus comparison is not discussed here.

Titanium dioxide $\left(\mathrm{TiO}_{2}\right)$ is taken as the particulate species and the gas phase mechanism of West et al. $[56,57]$ is used, although simplified artificial rates are used for easier analysis of the model behaviour. The $\mathrm{TiO}_{2}$ system is of industrial interest; however modelling efforts are hindered by the computational cost of high process rates under industrially relevant conditions. The performance is assessed by comparative convergence behaviour (the double 
type space should not affect the solution since the particle-number indices fully encode the particle space at the level of primary particles defined by monomer count), solver time savings, and reduction in required ensemble size.

Test cases

Two test cases are considered, a batch reactor and a continuously stirred tank reactor (CSTR) with no particles in the inflow. A spherical particle model is used in the first case and a detailed model is used in the second case. Both reactors are constant volume, at $1200 \mathrm{~K}$ and 4 bar (absolute). Their residence times are $6 \mathrm{~ms}$ and $10 \mathrm{~ms}$ respectively. Time steps of $0.01 \mathrm{~ms}$ and $0.1 \mathrm{~ms}$ are used respectively, with 10 splitting steps per step (convergence with decreasing splitting step was studied by Shekar et al. [16]).

A constant inception rate is used, with the inception particle size taken to be $0.49 \mathrm{~nm}\left(2 \mathrm{TiO}_{2}\right.$ units). Thus the particle-number model will always have zero particles at index 1 . In the first case, the coagulation rate is constant $K=\tilde{K}$, and in the second case, a transition regime coagulation kernel $K=$ $K^{\mathrm{tr}}$ is used (Appendix A). In both cases, sintering of neighbouring primary particles is not considered - note that the particle-number model does not introduce an an assumption of instantaneous sintering because in the current studies all coagulation events involving the particle-number particles transfer them to the discrete particle ensemble. The surface growth reaction adds $\mathrm{TiO}_{2}$ units to the particle surface and the rate depends on surface area only, 


$$
\beta_{\mathrm{SG}}\left(P_{i}\right)=\frac{\tilde{\beta}}{N_{\mathrm{A}}} \cdot A\left(P_{i}\right), \forall\left(P_{i}\right) \in \mathcal{M} \cup \mathcal{X}
$$

$$
\sigma_{\xi}^{\left(N_{\max }, L\right)}\left(t_{k}\right)=\sqrt{\frac{1}{L-1} \sum_{l=1}^{L}\left(\xi^{\left(N_{\max }, l\right)}\left(t_{k}\right)\right)^{2}-\left(\mu_{\xi}^{\left(N_{\max }, L\right)}\left(t_{k}\right)\right)^{2}} .
$$

390 The relative statistical error (Eq. (11)) is used to assess the random error 391 in repeat simulations at a given confidence level (99\% used here, with $\alpha_{0.99}$ 392 from the t-distribution).

$$
\bar{\epsilon}_{\text {stat }, \xi}^{\left(N_{\max }, L\right)}\left(t_{k}\right)=\frac{\alpha_{0.99}}{\sqrt{L-1}} \cdot \frac{\sigma_{\xi}^{\left(N_{\max }, L\right)}\left(t_{k}\right)}{\mu_{\xi}^{\left(N_{\max }, L\right)}\left(t_{k}\right)}
$$

393 The average relative total error (Eq. (12)) is used to assess the relative 
difference compared to a true solution $\xi^{*}$. Here, the 'true' solution is approximated by the solution with $N_{\max }=2^{18}$ and $L=10$ and the convergence study is performed for $N_{\max } \in\left\{2^{5}, 2^{6}, 2^{7}, \ldots, 2^{17}\right\}$, with $N_{\max } \times L=2^{18}$.

$$
\bar{\epsilon}_{\text {total }, \xi}^{\left(N_{\max }, L\right)}=\frac{1}{M} \sum_{k=1}^{M} \frac{\left|\mu_{\xi}^{\left(N_{\max }, L\right)}\left(t_{k}\right)-\xi^{*}\left(t_{k}\right)\right|}{\xi^{*}\left(t_{k}\right)}
$$

997 The properties used to illustrate convergence behaviour in this work include particle number concentration, $M_{0}(t)$ (Eq. (13)) and the average particle collision diameter, $d_{\mathrm{c}}$ (Eq. (14)) which is a measure of average particle size and is an example of a property that is of importance in applications.

$$
\begin{gathered}
M_{0}(t)=\frac{N\left(z_{\mathcal{M}}(t)\right)+N\left(z_{\mathcal{X}}(t)\right)}{V_{\mathrm{smp}}} \\
d_{\mathrm{c}}\left(P_{i}\right)=\frac{6 V_{i}}{A_{i}}\left(N_{\mathrm{pr}, i, i}\right)^{\frac{1}{1.8}}
\end{gathered}
$$

Solver time

Tests were run on one Intel Xeon E5-2640 CPU (2.40 GHz) of a 40 processor node with 200 GB RAM, running Red Hat Enterprise Linux version 7.2.

Case 1: constant rates batch reactor with spherical particle model

The constant rates case with spherical particle model is used to demonstrate proof of concept - under trivial constant rate conditions, the particle- 
number/particle model matches the convergence behaviour of the particle model (Figs. 6 and 7). The convergence tests were performed with $I=$ $10^{16} \mathrm{~cm}^{-3} \cdot \mathrm{s}^{-1}, \tilde{\beta}=10^{24} \mathrm{~cm}^{-5} \cdot \mathrm{s}^{-1}$ and $\tilde{K}=1.5 \times 10^{-15} \mathrm{~cm}^{-3} \cdot \mathrm{s}^{-1}$. A constant majorant kernel is used for coagulation and this has value $\hat{K}=1.5 \tilde{K}$.

The spherical particle model assumes each coagulation event is followed by instant coalescence to form a larger, spherical particle, so both type spaces hold the same information; however it should be possible to store/update this information more efficiently in a vector than a discrete ensemble. Surface growth events are performed once per particle since particles are not comprised of distinct primaries and choice of particles for coagulation and outflow is done by random selection (uniform selection criterion for Algorithm B.5). Thus the opportunities for improving run time with the PN/P model are limited; however, as expected it is more economical, especially for large ensembles (Table 1).

Case 2: transition kernel CSTR with detailed particle model

The transition coagulation kernel (Eq. (15)) is chosen because it is relevant to real synthesis conditions and depends on the properties of each particle which makes its evaluation more costly.

$$
K^{\mathrm{tr}}\left(P_{i}, P_{j}\right)=\frac{K^{\mathrm{sf}}\left(P_{i}, P_{j}\right) K^{\mathrm{fm}}\left(P_{i}, P_{j}\right)}{K^{\mathrm{sf}}\left(P_{i}, P_{j}\right)+K^{\mathrm{fm}}\left(P_{i}, P_{j}\right)}, \forall\left(P_{i}, P_{j}\right) \in \mathcal{M} \cup \mathcal{X}
$$

The transition regime coagulation kernel is found using the harmonic mean of the slipflow and free molecular kernels $\left(K^{\mathrm{sf}}, K^{\mathrm{fm}}\right)$. The slipflow kernel is sufficiently simple not to require a majorant kernel (Eq. (A.4)). 


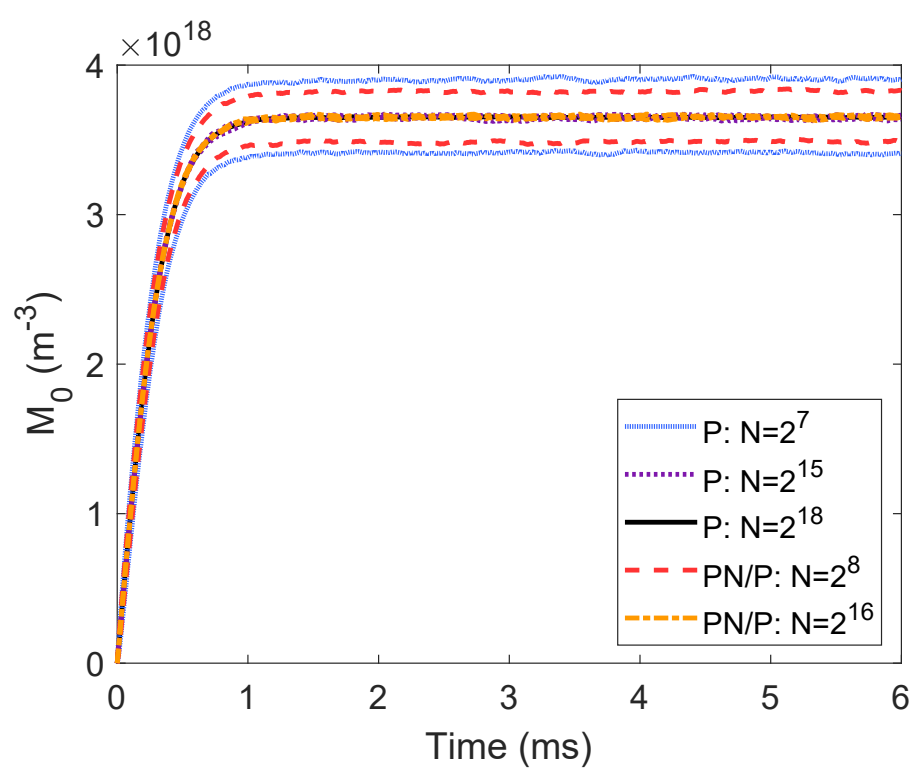

(a) Number density

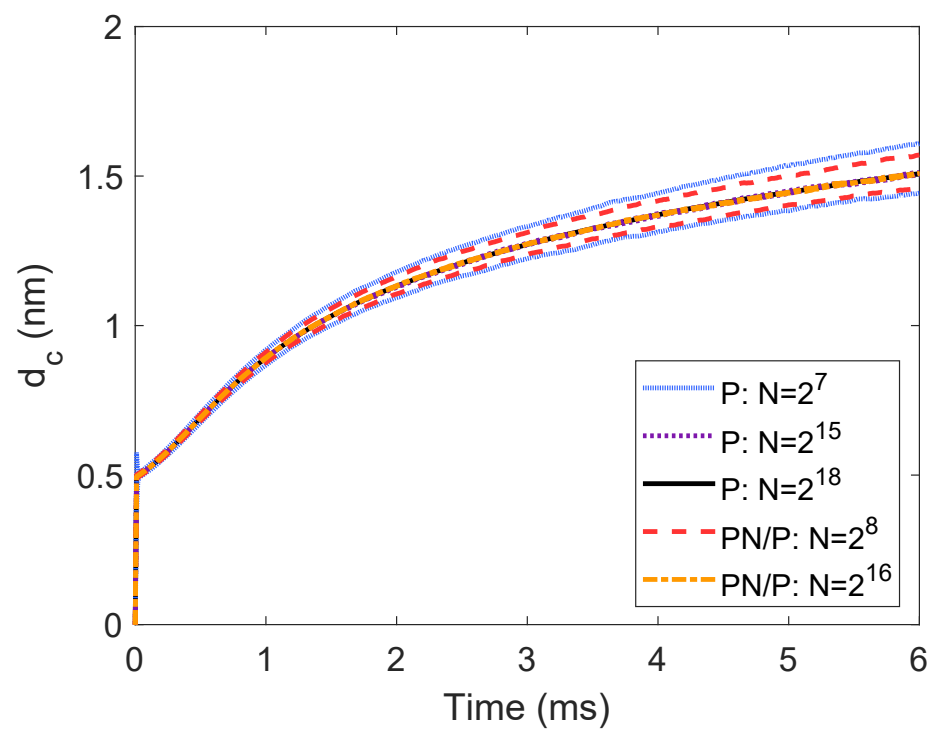

(b) Collision diameter

Figure 6: Transient properties in convergence study maintaining $N_{\max } \times L=2^{18}$ - the solid black line is the high fidelity solution and one standard deviation above and below the mean are shown as dotted lines for odd (particle model) and dashed lines for even (particle-number/particle model with $N_{\text {thresh }}=10^{2}$ ) powers of 2 (case 1$)$. 


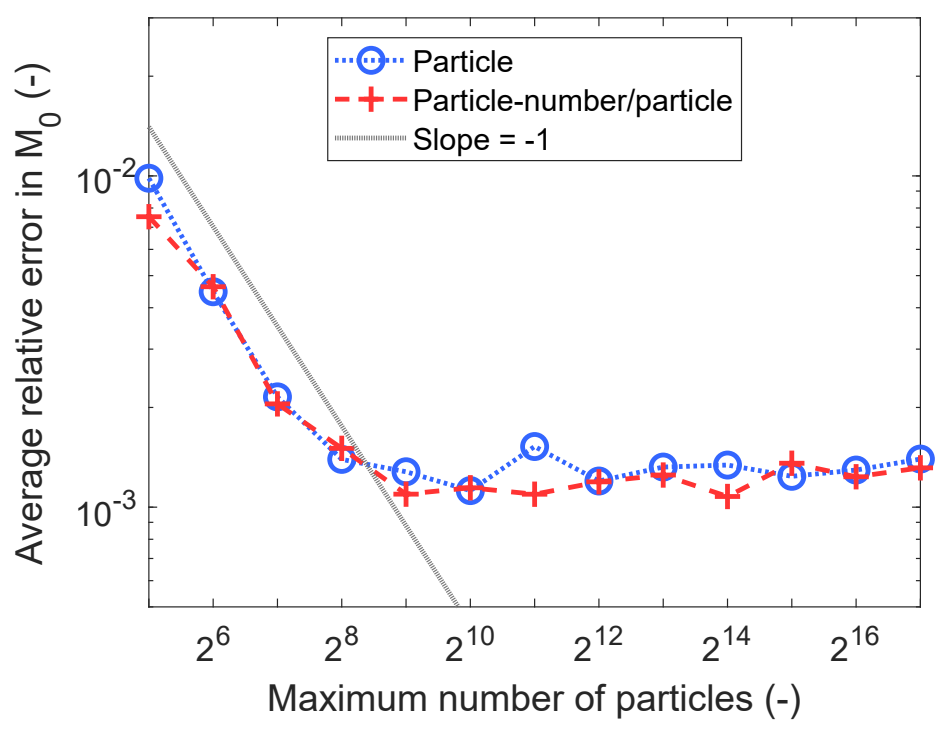

(a) Number density

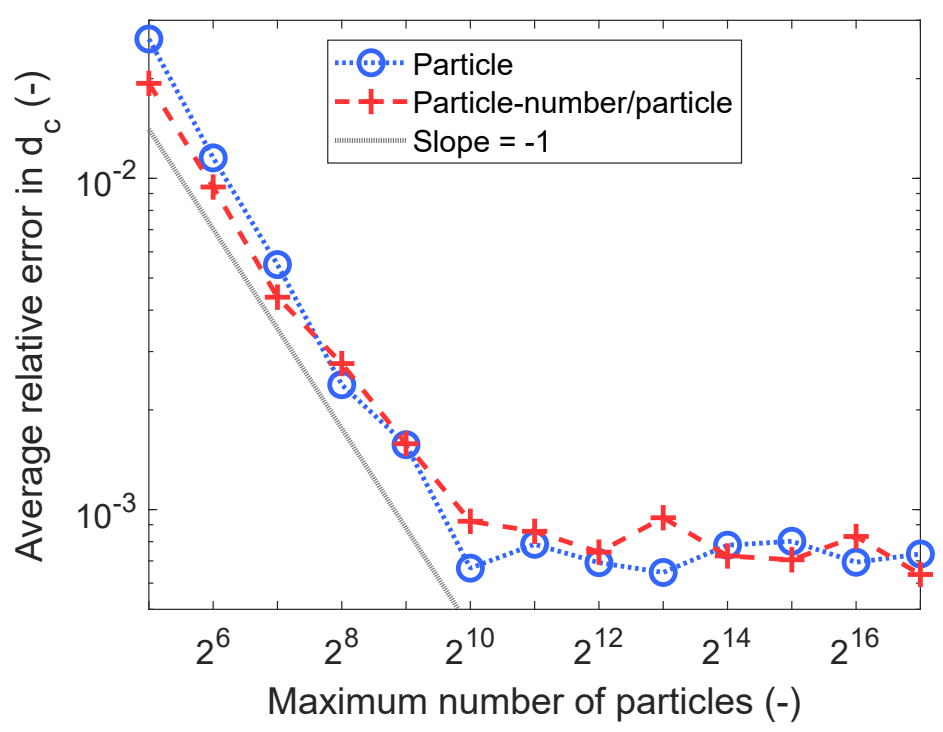

(b) Particle diameter

Figure 7: Convergence study maintaining $N_{\max } \times L=2^{18}$ - average relative total error (Eq. (12)) of the particle model and particle-number/particle model $\left(N_{\text {thresh }}=10^{2}\right)$ compared to the high fidelity solution (case 1 conditions). 
Table 1: Single run times for particle $(\mathrm{P})$ and particle-number/particle (PN/P) models with $N_{\text {thresh }}=10^{2}$ in the convergence study with case 1 conditions.

\begin{tabular}{cccc}
$\begin{array}{c}\text { Particles } \\
N_{\max }\end{array}$ & $\begin{array}{c}\text { Repeats } \\
L\end{array}$ & $\begin{array}{c}\text { Single run time } \\
\mathrm{P}(\min )\end{array}$ & $\begin{array}{c}\text { Single run time } \\
\text { PN/P }(\min )\end{array}$ \\
\hline $2^{7}$ & 2048 & 0.118 & 0.117 \\
$2^{8}$ & 1024 & 0.130 & 0.126 \\
$2^{9}$ & 512 & 0.154 & 0.143 \\
$2^{10}$ & 256 & 0.201 & 0.176 \\
$2^{11}$ & 128 & 0.336 & 0.265 \\
$2^{12}$ & 64 & 0.583 & 0.425 \\
$2^{13}$ & 32 & 1.18 & 0.797 \\
$2^{14}$ & 16 & 1.76 & 1.15 \\
$2^{15}$ & 8 & 3.06 & 1.94 \\
$2^{16}$ & 4 & 5.79 & 3.68 \\
$2^{17}$ & 2 & 12.3 & 7.99 \\
$2^{18}$ & 1 & 26.1 & 16.5 \\
\hline
\end{tabular}

A majorant for the free molecular kernel can be formed using inequalities for the nonlinear terms (Eq. (A.2)). This expression is useful because it does not require computation of the nonlinear terms for each particle pair to find the total rate. The rates for each kernel are split into several terms, computed as the sum of different particle properties across both type spaces, and these terms define particle selection rules used to choose a pair of particles (rates and selection rules in terms of particle properties are given in detail in Appendix A).

Surface growth is performed on every primary particle in each aggregate. The average relative error is compared with ten runs of the particle model with $N_{\max }=2^{18}$. The convergence tests were performed with $I=10^{12} \mathrm{~cm}^{-3}$. $\mathrm{s}^{-1}$ and $\tilde{\beta}=10^{24} \mathrm{~cm}^{-5} \cdot \mathrm{s}^{-1}$.

Here, the rates are more complicated, yet the simulation with the two 


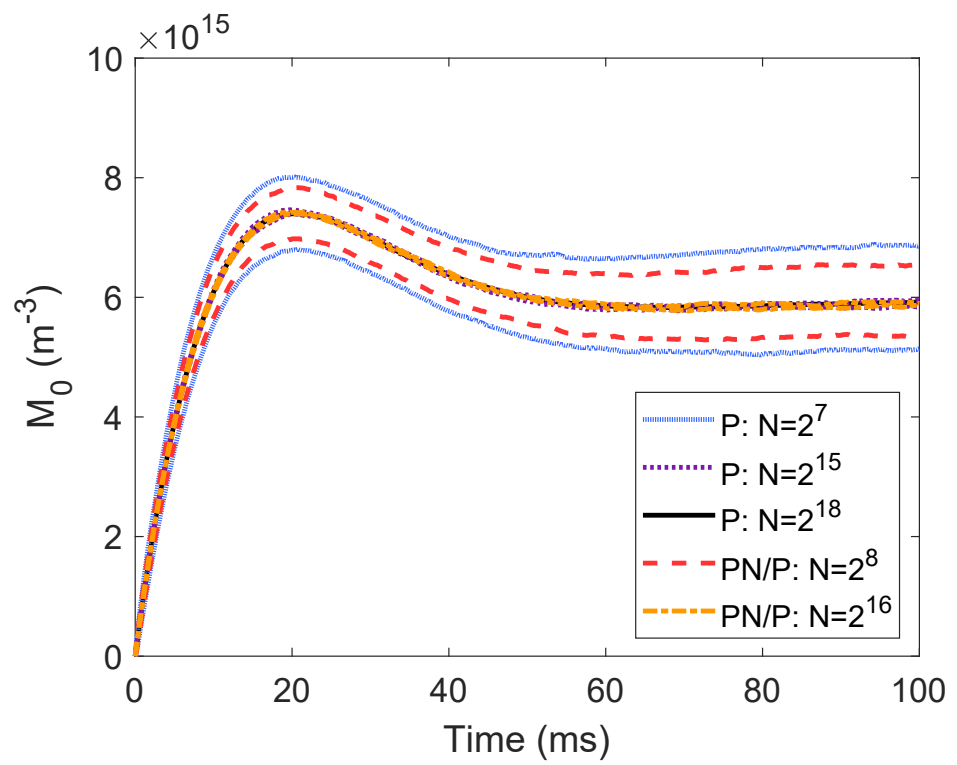

(a) Number density

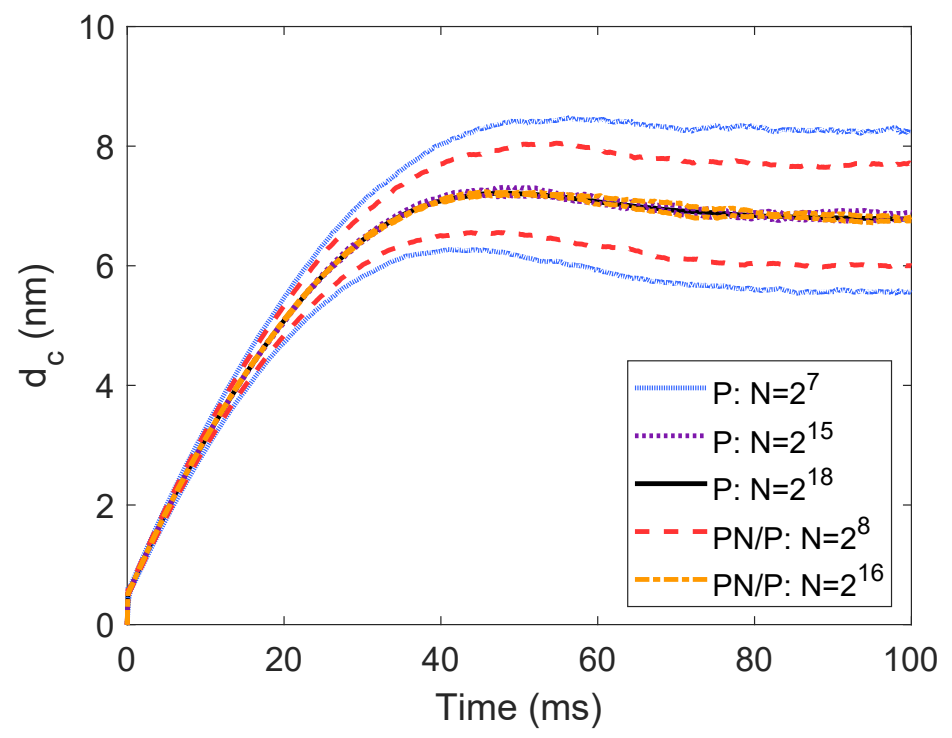

(b) Collision diameter

Figure 8: Transient properties in convergence study maintaining $N_{\max } \times L=2^{18}$ - the solid black line is the high fidelity solution and one standard deviation above and below the mean are shown as dotted lines for odd (particle model) and dashed lines for even (particle-number/particle model with $N_{\text {thresh }}=10^{4}$ ) powers of 2 (case 2 conditions). 


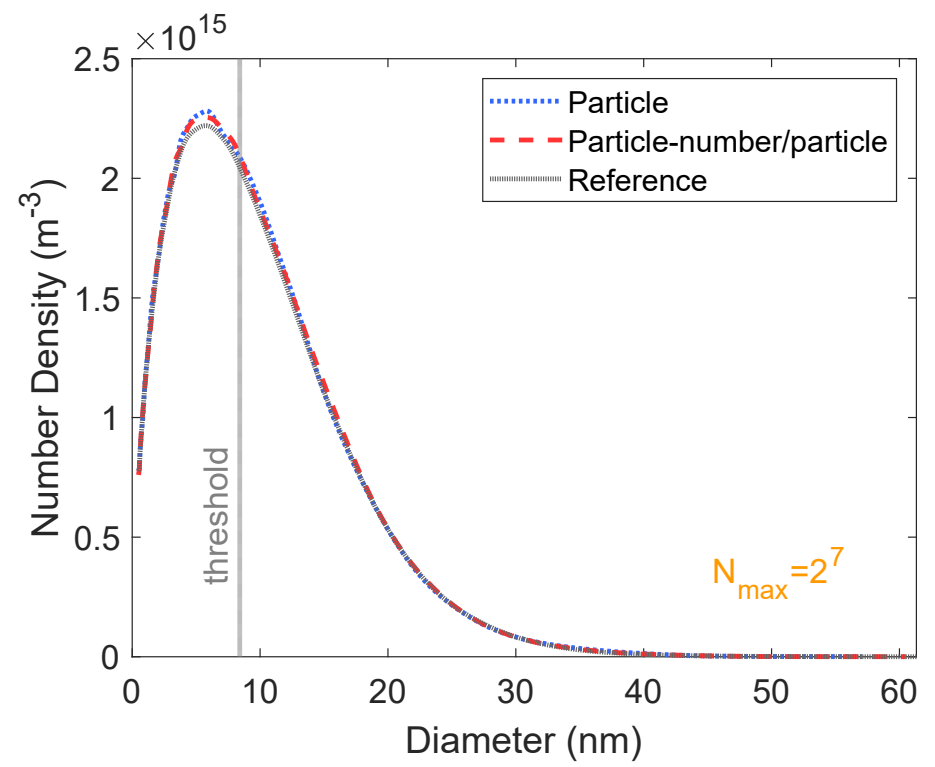

(a) $N_{\max }=2^{7}$



(b) $N_{\max }=2^{17}$

Figure 9: Kernel density estimates (bandwidth 0.07 ) for primary particle size distributions from particle model and particle-number/particle model with $N_{\text {thresh }}=10^{4}$ compared with reference solution with $N_{\max }=2^{18}$ and $L=10$. 


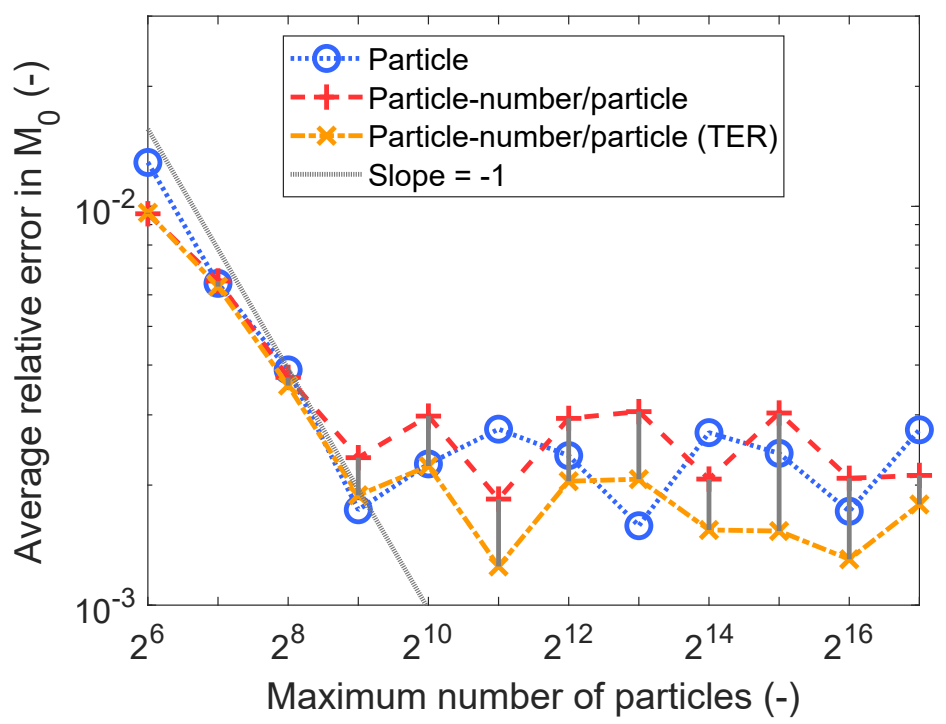

(a) Number density

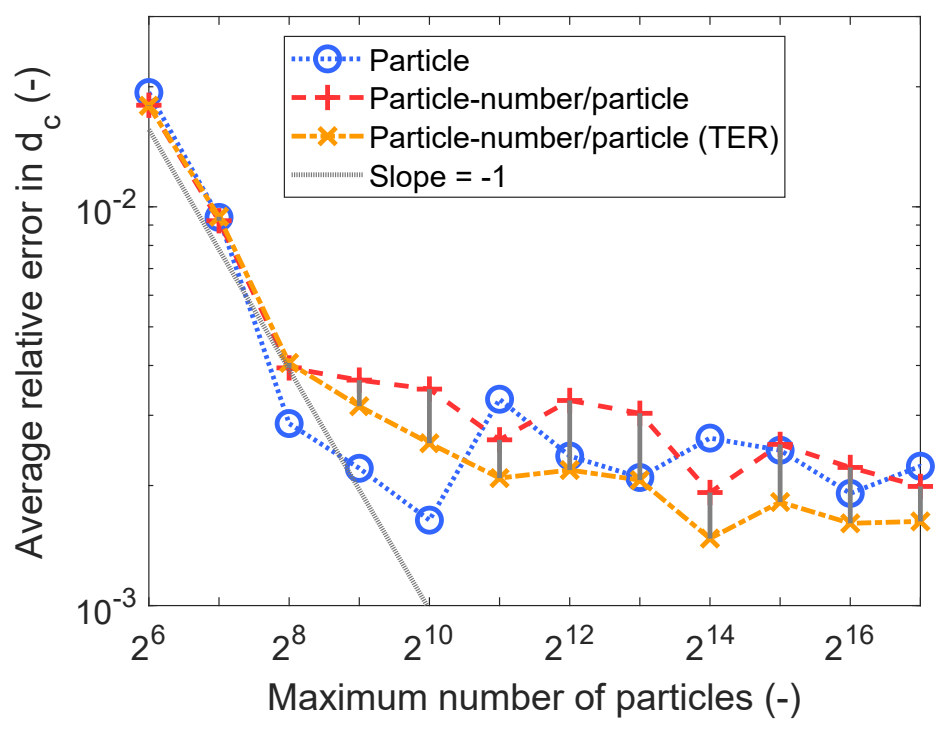

(b) Particle diameter

Figure 10: Convergence study maintaining $N_{\max } \times L=2^{18}$ - average relative total error (Eq. (12)) of the particle model, particle-number/particle model $\left(N_{\text {thresh }}=10^{4}\right)$, and $\mathrm{PN} / \mathrm{P}$ model with time equivalent runs (TER) compared to the high fidelity solution (case 2 conditions). 
type space models converges on the same properties as the single type space approach (Figs. 8-10); slight discrepancies between the PN/P model and the 'true' solution with the particle model may exist due to differences in the ordering of particles (i.e. a list in increasing size order vs. an unordered list of particles as formed could influence which particle is selected in Algorithm B.5); however, it is clear from the comparison of the steady-state particle size distributions (Fig. 9) that the algorithm for the PN/P model finds the same solution.

Differences in run time (Fig. 11) are more significant than in the study with the spherical particle model. This is especially noticeable for large ensembles where updates to the particle-number list are much more efficient than updates to distinct particles and a speed up of approximately $50 \%$ is observed for the ensembles with greater than $10^{5}$ particles. For small ensembles, the $\mathrm{PN} / \mathrm{P}$ model is more efficient in a narrower range of threshold values. In general, a threshold of $N_{\text {thresh }}=10^{4}$ was found to work well for the current conditions.

The reduced solver time is advantageous if CPU time is constrained; however the main benefit is that this allows an increase in the sample volume in the $\mathrm{PN} / \mathrm{P}$ model, i.e. use of a time equivalent sample volume (TESV, Table 2 column 5), or an increase in the number of repeat runs in the $\mathrm{PN} / \mathrm{P}$ model, i.e. use of time equivalent runs (TER, Table 2 column 6), to gain additional accuracy for comparable CPU cost (Fig. 10, solid vertical lines illustrate reduced error with additional repeats for same computational cost). The TESV is found by simulation: it is the sample volume for which the average run time of the $\mathrm{PN} / \mathrm{P}$ model matches that of the particle model. 


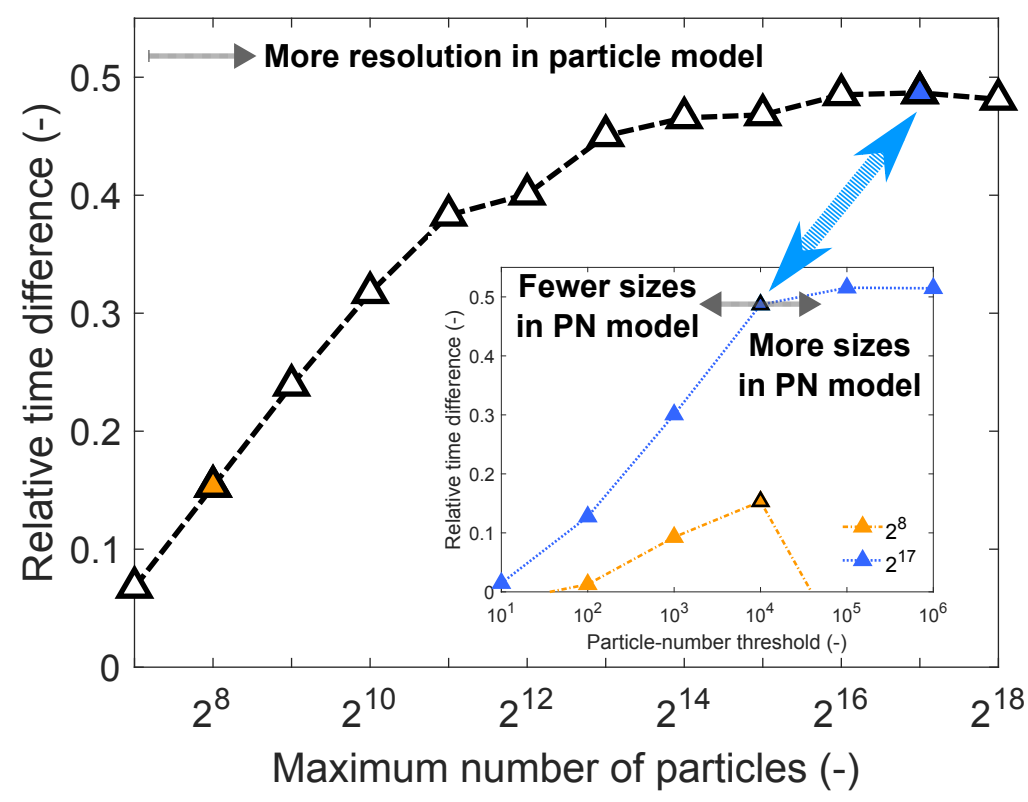

Figure 11: Relative time difference maintaining $N_{\max } \times L=2^{18}$ for pure particle model and particle-number(PN)/particle model with inset showing effect of threshold value $N_{\text {thresh }}$ (case 2 conditions).

The number of time equivalent runs $\left(L^{\mathrm{TER}}\right)$ is computed using the ratio of the average solver times $(\bar{t})$ for the particle and particle-number/particle simulations (Eq. (16)).

$$
L^{\mathrm{TER}}=\frac{\bar{t}_{\mathrm{P}}}{\bar{t}_{\mathrm{PN} / \mathrm{P}}} \cdot L
$$

The PN/P model removes most of the solo primary particles from the discrete particle ensemble, which allows the discrete ensemble to be used almost exclusively to resolve more complicated aggregate particles for the same computational cost and ensemble memory overhead by using a larger sample volume, as shown in the simulated imaging pictures in Fig. 12. This 
Table 2: Single run times, sample volume increase and additional repeats that can be achieved with solver time savings gained from PN/P model with $N_{\text {thresh }}=10^{4}$ (case 2 conditions).

\begin{tabular}{cccccc}
\hline $\begin{array}{c}\text { Particles } \\
N_{\max }\end{array}$ & $\begin{array}{c}\text { Repeats } \\
L\end{array}$ & $\begin{array}{c}\text { Single run time } \\
\mathrm{P}(\min )\end{array}$ & $\begin{array}{c}\text { Single run time } \\
\mathrm{PN} / \mathrm{P}(\mathrm{min})\end{array}$ & $\begin{array}{c}\text { TESV ratio } \\
V_{\text {smp }}^{\mathrm{TESV}} \cdot V_{\text {smp }}^{-1}\end{array}$ & $\begin{array}{c}\text { TER } \\
L^{\mathrm{TER}}\end{array}$ \\
\hline $2^{7}$ & 2048 & 0.339 & 0.316 & 1.67 & 2196 \\
$2^{8}$ & 1024 & 0.436 & 0.369 & 1.67 & 1209 \\
$2^{9}$ & 512 & 0.636 & 0.484 & 1.70 & 672 \\
$2^{10}$ & 256 & 1.05 & 0.717 & 1.74 & 375 \\
$2^{11}$ & 128 & 1.96 & 1.21 & 1.81 & 207 \\
$2^{12}$ & 64 & 3.46 & 2.07 & 1.88 & 107 \\
$2^{13}$ & 32 & 6.46 & 3.55 & 1.90 & 58 \\
$2^{14}$ & 16 & 9.23 & 4.93 & 1.95 & 30 \\
$2^{15}$ & 8 & 16.6 & 8.83 & 1.97 & 15 \\
$2^{16}$ & 4 & 31.3 & 16.1 & 2.00 & 8 \\
$2^{17}$ & 2 & 62.2 & 31.9 & 2.00 & 4 \\
$2^{18}$ & 1 & 124 & 64.6 & 2.03 & 2 \\
\hline
\end{tabular}

ensures that maximum utility is obtained from the detailed particle model without 'wasting' ensemble space and time on structurally simple particles. Increasing the sample volume increases the rate of numerical inceptions. The sample volume was chosen to ensure that the discrete ensemble never reached its maximum capacity in these studies, preventing random removals in all cases so that the statistical noise did not increase.

An alternative approach is to maintain a more economical memory footprint by initialising a smaller ensemble for tracking fewer distinct particles. This could be useful for systems that have an initial burst of particle inception due to high concentration of the gas phase precursor yielding a high initial number density. In such a system, doubling and contraction algorithms are often necessary with a discrete ensemble since demand for capacity varies 


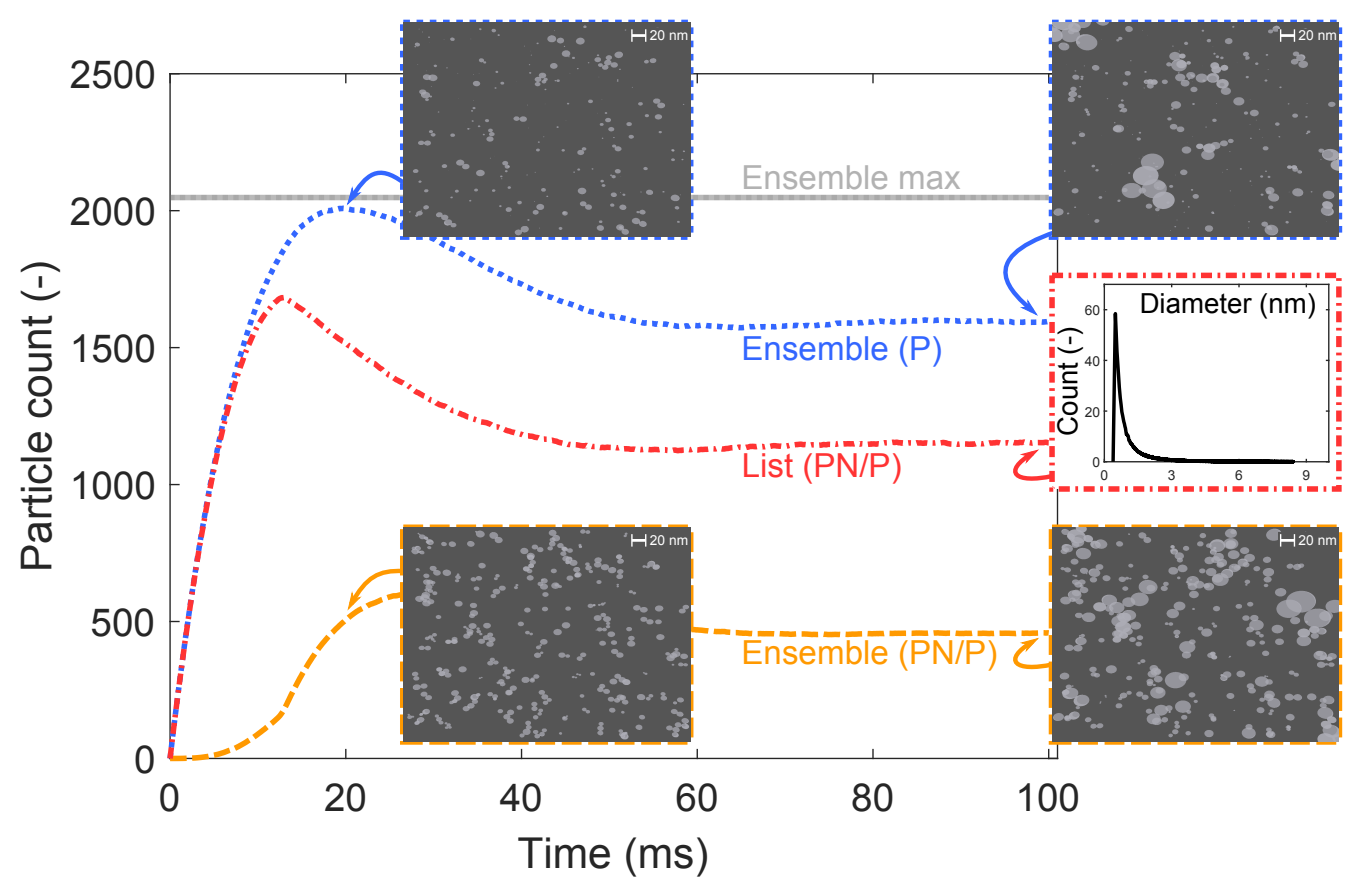

Figure 12: Particle counts in the ensemble and particle-number list for particle model (P) and particle-number/particle model (PN/P), with inset simulated SEMs of 200 tracked ensemble particles at $20 \mathrm{~ms}$ and $100 \mathrm{~ms}$ (scale bar shows $20 \mathrm{~nm}$ ) for $N_{\max }=2^{11}$ and $N_{\text {thresh }}=10^{4}$ (PN/P with runtime equivalent sample volume).

with time. The particle-number list can store arbitrarily many incepting particles so the ensemble can be customized to the size required to store aggregates only.

The effect of exceeding the ensemble capacity is illustrated further in Fig. 13. With a single discrete particle model, increasing the sample volume by a factor of three from the previous conditions results in contractions in the interval $t \in[4.8,20] \mathrm{ms}$ (shown in Fig. 13(a) with a horizontal arrow) because there is no space for new particles in the discrete ensemble so inceptions are accommodated by randomly removing an existing particle from the ensemble and scaling the sample volume to preserve the particle number density. With 
the hybrid type space model, particle inceptions contribute to the particlenumber space, $\mathcal{M}$, instead of being added to the ensemble space, $\mathcal{X}$. This list storage (shown in Fig. 13(a) with a vertical arrow) prevents the ensemble from flooding; thus no particles are removed.

Particle removal randomizes the system when the particles are polydisperse. This can be seen in Fig. 13(b): tripling the sample volume significantly increases the total error for the particle model ( $c f$. packed circle pattern labelled "P: $V_{\text {smp }}$ " and checkerboard pattern labelled "P: $3 V_{\text {smp }}$ ") whereas it reduces the total error for the hybrid model ( $c f$. wave pattern labelled "PN/P: $V_{\text {smp" }}$ and stripe pattern labelled "PN/P: $3 V_{\text {smp" }}$ ") due to the increased statistical significance of events in the larger sample volume.

\section{CSTR with particle inflow}

A second CSTR is added in series with the first using the conditions from case 2. The residence times are both $10 \mathrm{~ms}$, and the outflow from CSTR 1 is the only inflow stream to CSTR 2. This case demonstrates the use of the particle-number/particle inflow algorithm (Alg. B.3) as there are particles in the outflow from CSTR 1. The primary PSD shifts towards larger particles in CSTR 2 due to further surface growth (Fig. 14). This study also provides insight into the transient statistical error behaviour (Eq. (11)) in a flow reactor. As shown in previous work [42], the error increases before reaching a plateau as the system reaches steady state. The same sample volume was used for both reactors. For the second CSTR with the particle model, random removal events occurred from ca. $\tau_{\mathrm{CSTR} 2}$, reducing the sample volume (shown as a dashed black line in $15(\mathrm{~b})$ ). The sample volume in the second CSTR was constant for the particle-number model, due to use of the 


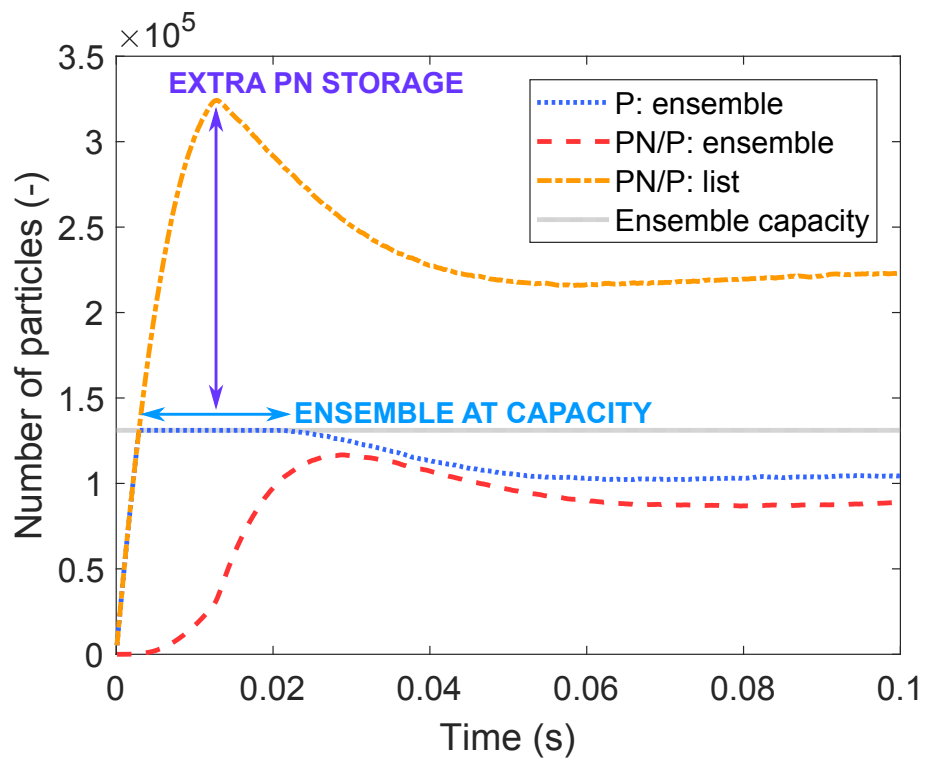

(a) Particle counts in $3 V_{\text {smp }}$ case

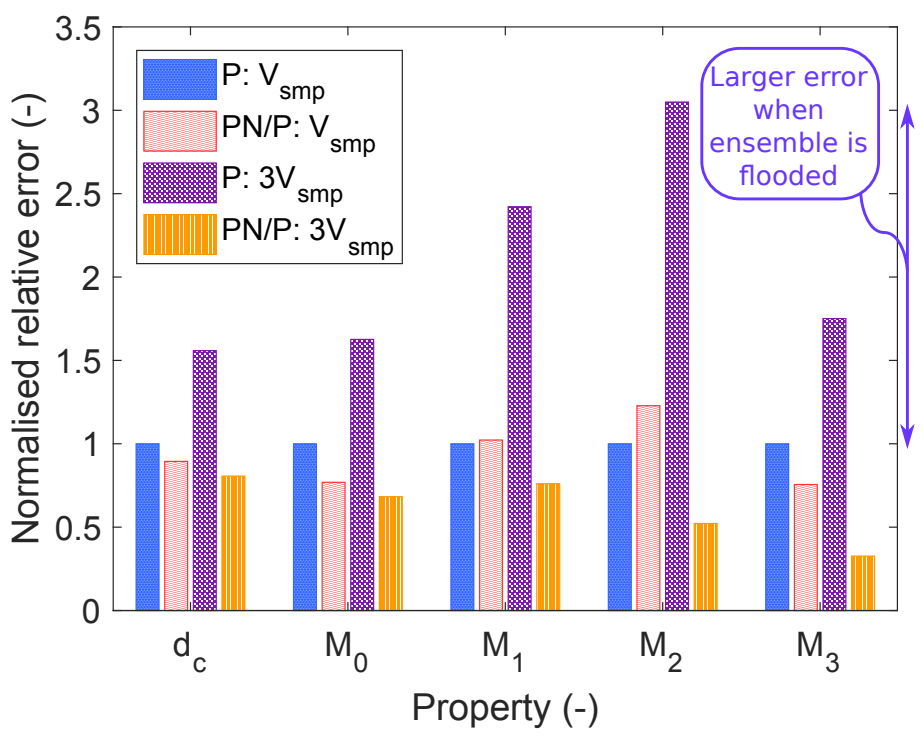

(b) Average error in converged solutions

Figure 13: Effect of exceeding ensemble capacity with $N_{\max }=2^{17}$ - normalised total relative error in: particle model; PN/P model $\left(N_{\text {thresh }}=10^{4}\right)$; particle model with triple sample volume; and PN/P model with triple sample volume (case 2 conditions). 


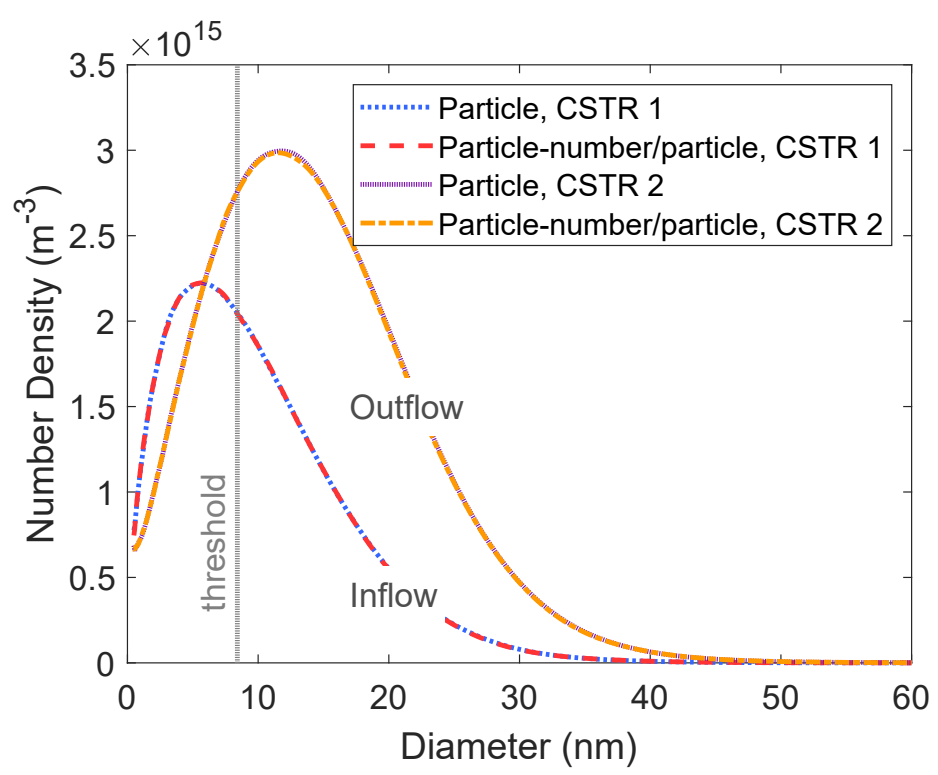

Figure 14: Steady state kernel density estimate of the primary particle size distribution in the inflow and outflow from CSTR 2 (bandwidth of 0.07 ), for the particle and particlenumber/particle $\left(N_{\text {thresh }}=10^{4}\right)$ models with $N_{\max }=2^{14}$ and $L=160$.

Table 3: Inception and surface reaction rate constants used in rate study.

\begin{tabular}{cccccc}
\hline Process & Units & \multicolumn{4}{c}{ Rate constants } \\
\hline Inception & {$\left[\mathrm{cm}^{-3} \cdot \mathrm{s}^{-1}\right]$} & $1 \times 10^{6}$ & $1 \times 10^{9}$ & $1 \times 10^{12}$ & $1 \times 10^{13}$ \\
Surface reaction & {$\left[\mathrm{cm}^{-5} \cdot \mathrm{s}^{-1}\right]$} & $1 \times 10^{18}$ & $1 \times 10^{21}$ & $1 \times 10^{24}$ & \\
\hline
\end{tabular}

particle-number list to store inflowing and incepting particles. Thus, the steady statistical error in the second CSTR was slightly lower (Fig. 15(b)).

\subsection{Performance of $P N / P$ model in different rate regimes}

Performance of the PN/P model is assessed in different rate regimes using the conditions in Table 3, for the CSTR from case 2 with a transition regime coagulation kernel and a detailed particle model for the aggregate type space.

The process rates are coupled since the coagulation rate increases quadrat- 


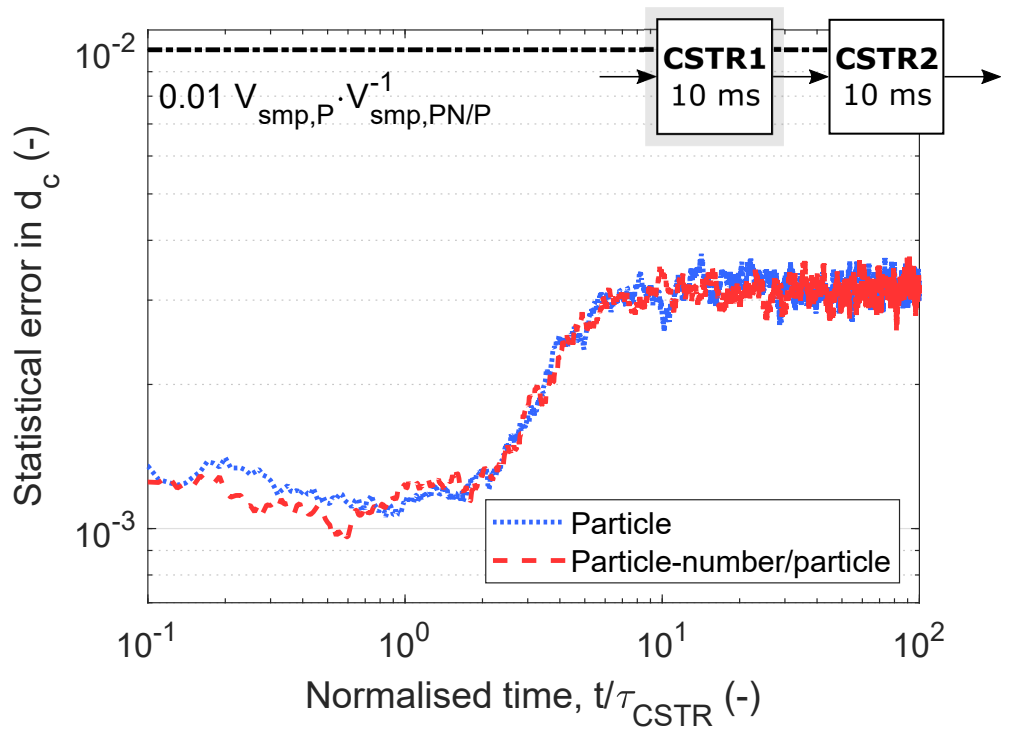

(a) CSTR 1

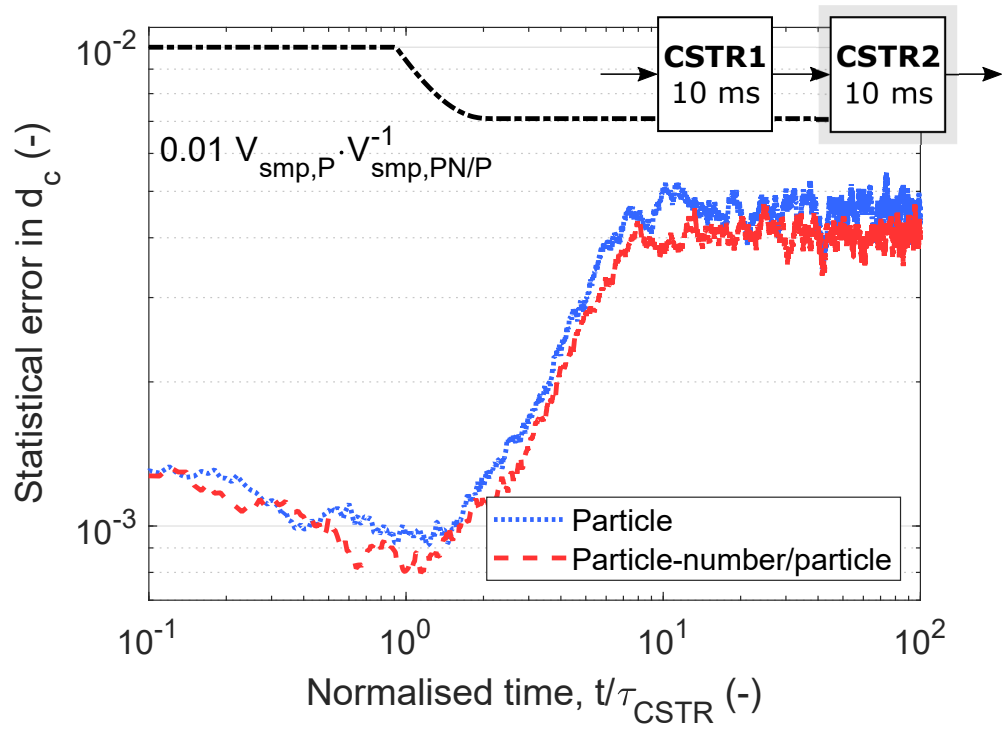

(b) CSTR 2

Figure 15: Transient statistical error at 99\% confidence level, using t-distribution values, in a pair of CSTRs connected in series, for the particle and particle-number/particle $\left(N_{\text {thresh }}=10^{4}\right)$ models with $N_{\max }=2^{14}$ and $L=160$. 
ically with number density and depends on properties of the particles such as diameter. To simplify the analysis, the average ratio of the rates is used in Figs. 16 and 18:

$$
\text { Mean rate ratio (inception:coagulation) }=\frac{1}{M} \sum_{m=1}^{M} \frac{R_{\text {inception }}\left(t_{m}\right)}{R_{\text {coagulation }}\left(t_{m}\right)}
$$

Mean rate ratio (surface reaction:coagulation) $=\frac{1}{M} \sum_{m=1}^{M} \frac{R_{\text {surface reaction }}\left(t_{m}\right)}{R_{\text {coagulation }}\left(t_{m}\right)}$.

The mean count ratio is used to assess the utility of the particle-number list for storing particles and refers to the average particle-number count divided by the average ensemble count:

$$
\text { Mean count ratio }=\frac{1}{M} \sum_{m=1}^{M} \frac{N\left(z_{\mathcal{M}}\left(t_{m}\right)\right)}{N\left(z_{\mathcal{X}}\left(t_{m}\right)\right)}
$$

The combined particle-number/(detailed)particle model offers considerable performance advantages over the use of a single detailed particle model for conditions that result in a large number of solo primary particles (when inception dominates coagulation). In these cases, most of the particles in the system can be stored in the particle-number list, significantly reducing the ensemble size requirements (Fig. 16). Conditions with high surface growth and similar coagulation and inception rates do not see significant solver time advantage with the PN/P model (Fig. 17) because the coagulation processes produce large aggregates and the surface updates for these complex structures dominate the solver time; however, there are still significantly many primary 
Transfer to ensemble by coagulation

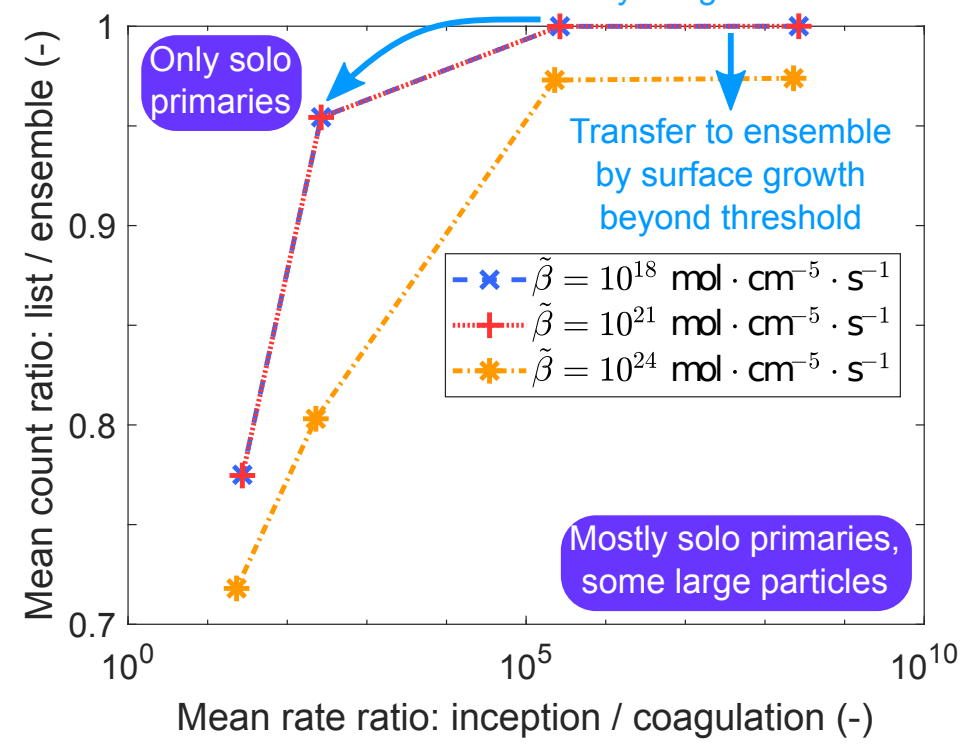

Figure 16: Ratio of particles in the particle-number list to particles in the ensemble in the $\mathrm{PN} / \mathrm{P}$ model for different ratios of inception rate to coagulation rate (using threshold $N_{\text {thresh }}=2^{17}$ ). 


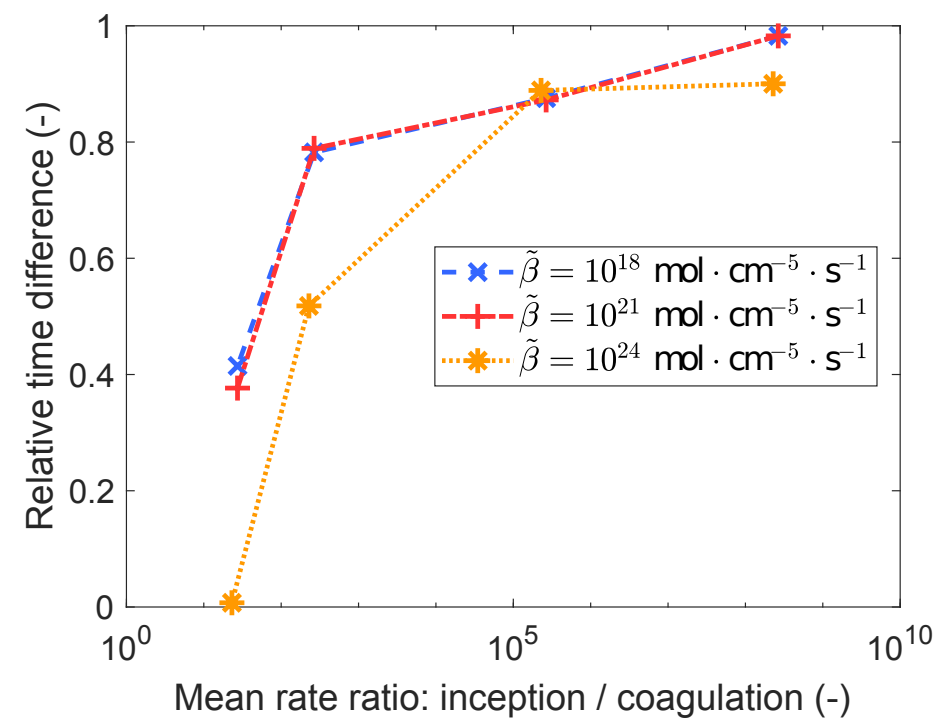

Figure 17: Solver time difference for different ratios of inception rate to coagulation rate (using threshold $N_{\text {thresh }}=2^{17}$ ).

particles in the particle-number list under these conditions and the option to use a smaller particle ensemble could still be attractive due to improved memory efficiency. Future work should consider methods for mitigating the aggregate update cost.

When the surface growth rate is very high, primary particles grow rapidly and are pulled out of the particle-number system into the particle system unless a large threshold value is used to store the primaries in the particlenumber system for as long as possible (Fig. 18). The number density of very large primaries becomes lower with increasing index (Fig. 19), so use of a high threshold (e.g. $N_{\text {thresh }}=10^{4}$ ) achieves limited additional particle storage; however, since the updates to the particle-number model are comparatively cheap even for large thresholds, it is reasonable to use a large threshold to 


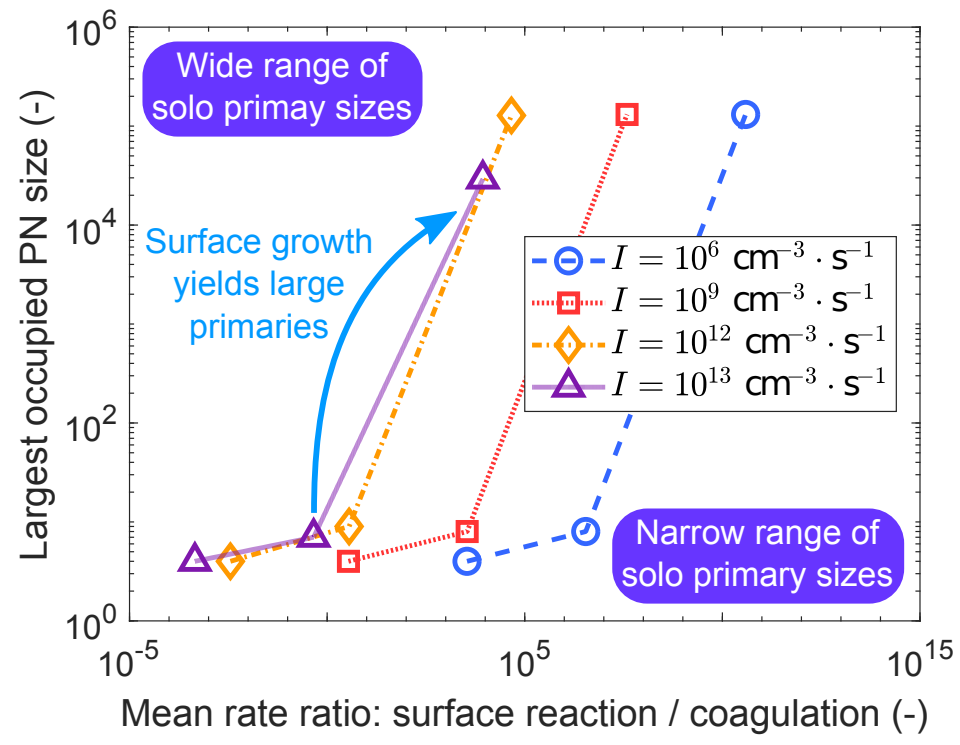

Figure 18: Largest occupied particle-number $(\mathrm{PN})$ size for different ratios of surface reaction rate to coagulation rate (using threshold $N_{\text {thresh }}=2^{17}$ ).

avoid wasting ensemble space on single primary particles.

\section{Conclusion}

This work proposes a stochastic population balance algorithm using a detailed particle model to resolve complex particles and a particle-number model for simple particles. This improves computational resolution of particles when the PSD is broad and aggregate particle morphology is important because arbitrarily many primary particles can be stored in the number list. We show that a larger sample volume can be tolerated for a given ensemble size, without causing random removal of particles. Because updating particles in the list only requires updating a counter, this approach is also more efficient in general. The improved efficiency is expected to be particularly 


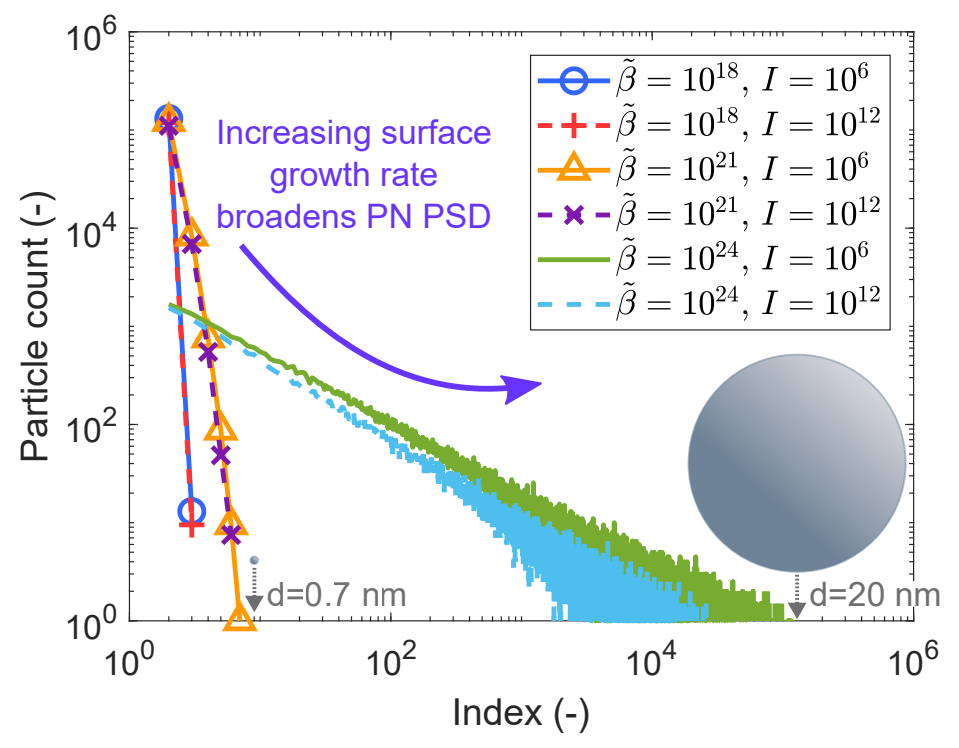

Figure 19: Particle-number (PN) size distributions at $t_{f}$ for different ratios of surface reaction rate to coagulation rate (using threshold $N_{\text {thresh }}=2^{17}$ ).

important under high concentration conditions, such as modelling industrial particle synthesis.

Under low surface growth conditions, the required threshold to store all primaries is small because the range of primary sizes is narrow; however, under high surface growth conditions, it could be advantageous to use a larger threshold in order to accommodate the wider range of primary sizes and benefit from the more efficient update structure of the particle-number list. The proposed hybrid model is less effective when the coagulation rate is very high, because the computational complexity associated with very large aggregate particles dominates the solver time. The hybrid scheme offers two main benefits.

1. It can be up to $50 \%$ faster than a single detailed particle type space 
model when the surface growth rate is high and the surface updates to ensemble particles are expensive. This speed-up can be traded for a larger sample volume to achieve a greater statistical accuracy for comparable cost and memory. One possible application where this would make a really significant improvement is if particle-particle heat transfer effects were included and the surface updates for each particle were even more costly.

2. When the inception/coagulation ratio is large, most particles can be stored in the particle-number list, reducing the size of particle ensemble required to resolve the aggregate particles. This smaller ensemble has a lower memory footprint. One possible application would be in coupling to computational fluid dynamics simulations where the memory and computational cost associated with large ensembles would be prohibitive. This also assists tailoring the ensemble to the size needed to store aggregate particles, by avoiding initial periods of high inception when the precursor concentration is high, without resorting to contraction and doubling algorithms.

A number of adaptations are possible for different systems.

1. If the internal co-ordinate is not 'quantized' (multiples of a monomer subunit), the indexing can be converted to sections of larger width at the cost of introducing some approximation error within the sections.

2. For more efficiency, it might be assumed that collisions between small particles result in instant coalescence, allowing these collisions to be performed in the particle-number model. This could be controlled using 
the sintering rate to determine where this assumption is near to the actual behaviour.

3. Weighted particle methods such as described by Patterson et al. [47] could be employed to reduce the number of particles injected to the ensemble by surface growth beyond the threshold.

\section{Acknowledgements}

This project is partly funded by the National Research Foundation (NRF), Prime Minister's Office, Singapore under its Campus for Research Excellence and Technological Enterprise (CREATE) programme. The authors would also like to thank Venator for financial support. 
${ }_{613}$ Nomenclature

\section{Upper-case Roman}

$A$ Surface area

$\left[\mathrm{m}^{2}\right]$

C Concentration

$\left[\mathrm{mol} \cdot \mathrm{m}^{-3}\right]$

$F \quad$ Ratio

$I$ Inception rate

$\left[\mathrm{mol} \cdot \mathrm{m}^{-3} \cdot \mathrm{s}^{-1}\right]$

$K$ General coagulation kernel

$\left[\mathrm{m}^{-3} \cdot \mathrm{s}^{-1}\right]$

$\tilde{K}$ Coagulation constant

$\hat{K} \quad$ Majorant coagulation kernel

Kn Knudsen number

${ }^{614} L$ Number of repeat runs

$M \quad$ Number of time steps

$M_{0} \quad 0^{\text {th }}$ number moment

$\left[\mathrm{m}^{-3}\right]$

$N$ Number

$N_{\text {A }}$ Avogadro's constant

$\left[\mathrm{mol}^{-1}\right]$

P Particle

Poi Poisson distribution

$R$ Rate

[process specific]

$T$ Temperature

$[\mathrm{K}]$

$U$ Uniform distribution

V Volume

$\left[\mathrm{m}^{3}\right]$ 


\section{Lower-case Roman}

c Constant

d Diameter

$[\mathrm{nm}]$

$f \quad$ Volumetric feed fraction

$g$ Surface growth type-change function

$k_{\mathrm{B}}$ Boltzmann constant

$m$ Mass

$n$ Particle number concentration

$p$ Primary particle

$t$ Time

$x \quad$ Particle type variable

y Particle type variable

z Particle system

\section{Lower-case Greek}

$\alpha$ Random variable

$\beta$ Surface growth rate

$\left[\mathrm{m}^{2} \cdot \mathrm{m}^{-3} \cdot \mathrm{s}^{-1}\right]$

$\tilde{\beta}$ Surface growth constant

$\gamma$ Weighted random variable

$\bar{\epsilon}$ Average relative error

$\mu$ Viscosity

$[\mathrm{Pa} \cdot \mathrm{s}]$ 
$\mu_{\xi} \quad$ Mean value of property $\xi$

$\xi$ Property

$\rho$ Mass density

$\left[\mathrm{kg} \cdot \mathrm{m}^{-3}\right]$

$\sigma_{\xi} \quad$ Standard deviation of property $\xi$

$\tau$ Residence time

$[\mathrm{S}]$

$\phi \quad$ Arbitrary continuous function

\section{Superscripts}

$\begin{aligned} \text { fm } & \text { Free molecular } \\ \text { in } & \text { inflow } \\ \text { out } & \text { Outflow } \\ \text { sf } & \text { Slip flow } \\ \text { tr } & \text { Transition } \\ * & \text { Denotes reference solution }\end{aligned}$

\section{Subscripts}

$\begin{aligned} \text { c } & \text { Collision } \\ \text { coag } & \text { Coagulation } \\ i & \text { Index variable } \\ \text { in } & \text { inflow } \\ \text { inc } & \text { inception } \\ j & \text { Index variable }\end{aligned}$




$\begin{aligned} k & \text { Index variable } \\ \text { max } & \text { Maximum } \\ \text { out } & \text { Outflow } \\ \text { pri } & \text { Primary particle } \\ \text { SG } & \text { Surface growth } \\ \text { smp } & \text { Sample } \\ \text { split } & \text { Splitting time } \\ \text { stat } & \text { Statistical } \\ \text { thresh } & \text { Threshold } \\ \text { tmp } & \text { Template } \\ 1 & \text { Denotes monomer size (first) index }\end{aligned}$

\section{Symbols}

$\mathcal{E}$ Generic particle type space

$\mathcal{F}$ Flow operator

$\mathcal{K}$ Coagulation operator

$\mathcal{I}$ Inception operator

$\mathcal{M}$ Small particle type space

$\mathcal{P}$ Pressure

$\mathbb{P}$ Mathematical probability

$\mathcal{S}$ Surface growth operator

$\mathcal{X}$ Large particle type space 
1 Indicator function

$\forall \quad$ For all

\section{Abbreviations}

CFD Computational fluid dynamics

CSTR Continuous stirred tank reactor

DSA Direct simulation algorithm

DQMOM Direct quadrature method of moments

LPDA Linear process deferment algorithm

618

MOMIC Method of moments with interpolative closure

ODE Ordinary differential equation

PBE Population balance equation

PN/P Particle-number/particle

PSD Particle size distribution

DQMOM Direct quadrature method of moments

QMOM Quadrature method of moments

SWA Stochastic weighted algorithm

SEM Scanning electron microscopy

TER Time-equivalent repeats

TESV Time-equivalent sample volume 


\section{Appendix A. Transition regime coagulation kernel}

The transition kernel has the form

$$
K^{\mathrm{tr}}\left(P_{i}, P_{j}\right)=\frac{K^{\mathrm{sf}}\left(P_{i}, P_{j}\right) K^{\mathrm{fm}}\left(P_{i}, P_{j}\right)}{K^{\mathrm{sf}}\left(P_{i}, P_{j}\right)+K^{\mathrm{fm}}\left(P_{i}, P_{j}\right)}, \forall\left(P_{i}, P_{j}\right) \in \mathcal{M} \cup \mathcal{X},
$$

where $K^{\text {sf }}$ and $K^{\text {fm }}$ are the slip-flow and free-molecular kernels defined below in which in which $m$ is the particle mass, $k_{\mathrm{B}}$ is the Boltzmann constant, $\mathcal{P}$ is the pressure, and $\mathrm{Kn}$ is the Knudsen number [16].

$$
\begin{aligned}
K_{\mathrm{sf}}\left(P_{i}, P_{j}\right) & =\frac{2 k_{\mathrm{B}} T}{3 \mu}\left(\frac{1+1.257 \mathrm{Kn}\left(P_{i}\right)}{d_{\mathrm{c}}\left(P_{i}\right)}+\frac{1+1.257 \mathrm{Kn}\left(P_{j}\right)}{d_{\mathrm{c}}\left(P_{j}\right)}\right)\left(d_{\mathrm{c}}\left(P_{i}\right)+d_{\mathrm{c}}\left(P_{j}\right)\right) \\
K_{\mathrm{fm}}\left(P_{i}, P_{j}\right) & =2.2 \sqrt{\frac{\pi k_{\mathrm{B}} T}{2}\left(\frac{1}{m\left(P_{i}\right)}+\frac{1}{m\left(P_{j}\right)}\right)}\left(d_{\mathrm{c}}\left(P_{i}\right)+d_{\mathrm{c}}\left(P_{j}\right)\right)^{2} \\
\mathrm{Kn}\left(P_{i}\right) & =4.74 \times 10^{-8} \frac{T}{\mathcal{P} d_{\mathrm{c}}\left(P_{i}\right)}
\end{aligned}
$$

Majorant kernel techniques are used to reduce the computational complexity of evaluating the double summation over the particle space for the non-linear coagulation kernel. The technique used here is described by Patterson et al. [47] and Menz et al. [58]. The kernel $K$ is bounded by a larger kernel $\hat{K}$ which is easier to evaluate. In order to achieve the correct coagulation behaviour, the majorant rate is used to compute the total coagulation rate $R_{\text {coag }}(2)$; however individual coagulation events between particles $P_{i}$ and $P_{j}$ are only performed with probability $K_{i j} \cdot \hat{K}_{i j}^{-1}$.

The majorant used for the free-molecular kernel is 


$$
\hat{K}_{\mathrm{fm}}\left(P_{i}, P_{j}\right)=4.4 \sqrt{\frac{\pi k_{\mathrm{B}} T}{2}}\left(\frac{1}{\sqrt{m\left(P_{i}\right)}}+\frac{1}{\sqrt{m\left(P_{j}\right)}}\right)\left(d_{\mathrm{c}}\left(P_{i}\right)^{2}+d_{\mathrm{c}}\left(P_{j}\right)^{2}\right) .
$$

633 Define

$$
\beta_{1}=4.4 \sqrt{\frac{\pi k_{\mathrm{B}} T}{2}}
$$

634 Then

$$
\hat{K}_{\mathrm{fm}}\left(P_{i}, P_{j}\right)=\beta_{1}\left(\frac{d_{\mathrm{c}}\left(P_{i}\right)^{2}}{\sqrt{m\left(P_{i}\right)}}+\frac{d_{\mathrm{c}}\left(P_{i}\right)^{2}}{\sqrt{m\left(P_{j}\right)}}+\frac{d_{\mathrm{c}}\left(P_{j}\right)^{2}}{\sqrt{m\left(P_{i}\right)}}+\frac{d_{\mathrm{c}}\left(P_{j}\right)^{2}}{\sqrt{m\left(P_{j}\right)}}\right) .
$$

The slip-flow kernel does not require a majorant. Define

$$
\begin{aligned}
& \beta_{2}=\frac{2 k_{\mathrm{B}} T}{3 \mu} \\
& \beta_{3}=1.257 \times 4.74 \times 10^{-8} \frac{T}{\mathcal{P}} .
\end{aligned}
$$

636

Then

$K_{\mathrm{sf}}\left(P_{i}, P_{j}\right)=\beta_{2}\left(2+\frac{d_{\mathrm{c}}\left(P_{i}\right)}{d_{\mathrm{c}}\left(P_{j}\right)}+\frac{d_{\mathrm{c}}\left(P_{j}\right)}{d_{\mathrm{c}}\left(P_{i}\right)}+\beta_{3}\left(\frac{1}{d_{\mathrm{c}}\left(P_{i}\right)}+\frac{d_{\mathrm{c}}\left(P_{i}\right)}{d_{\mathrm{c}}\left(P_{j}\right)^{2}}+\frac{d_{\mathrm{c}}\left(P_{j}\right)}{d_{\mathrm{c}}\left(P_{i}\right)^{2}}+\frac{1}{d_{\mathrm{c}}\left(P_{j}\right)}\right)\right)$. 
Table A.4: Particle properties used to choose coagulation pair $\left(P_{i}, P_{j}\right)$ based on transition regime majorant kernel terms.

\begin{tabular}{cccc}
\hline Term & Equation & $P_{i}$ & $P_{j}$ \\
\hline Free-molecular 1 & $(N(t)-1) \sum d_{i}^{2} m_{i}^{-1 / 2}$ & Uniform & $d_{\mathrm{c}}\left(P_{j}\right)^{2} \cdot m\left(P_{j}\right)^{-0.5}$ \\
Free-molecular 2 & $\sum d_{i}^{2} \sum m_{i}^{-1 / 2}-\sum d_{i}^{2} m_{i}^{-1 / 2}$ & $d_{\mathrm{c}}\left(P_{i}\right)^{2}$ & $m\left(P_{j}\right)^{-0.5}$ \\
Slip-flow 1 & $N(t)(N(t)-1)$ & Uniform & Uniform \\
Slip-flow 2 & $\sum d_{i} \sum d_{i}^{-1}-N(t)$ & $d_{\mathrm{c}}\left(P_{i}\right)$ & $d_{\mathrm{c}}\left(P_{j}\right)^{-1}$ \\
Slip-flow 3 & $(N(t)-1) \sum d_{i}^{-1}$ & Uniform & $d_{\mathrm{c}}\left(P_{j}\right)^{-1}$ \\
Slip-flow 4 & $\sum d_{i} \sum d_{i}^{-2}-\sum d_{i}^{-1}$ & $d_{\mathrm{c}}\left(P_{i}\right)$ & $d_{\mathrm{c}}\left(P_{j}\right)^{-2}$ \\
\hline
\end{tabular}

By the techniques described in Patterson et al. [47], this yields the equations and selection properties given in Table A.4 for coagulation rate terms and particle pairs respectively. Particles are chosen for coagulation events according to individual property-dependent rates (Table A.4). The six selection probabilities in the third and fourth columns of Table A.4 are specified by the corresponding coagulation rate terms in the second column. The rate terms arise from summation of the majorant kernel over all particles. These are used to define probabilities of each selection process being chosen for a coagulation event. Once a process is selected, the corresponding selection probabilities are used to choose a particle pair (that is, the particle property $\xi$ in the selection algorithm, B.5, is specified by the relevant row and column of Table A.4). Thus, the particle particle pairs with higher majorant rates are selected more often than the ones with lower rates. The real coagulation rate for the coagulating particle pair is compared to its majorant rate and this defines the probability of a real/fictitious event (Eq. (4)). 


\section{Appendix B. Algorithms}

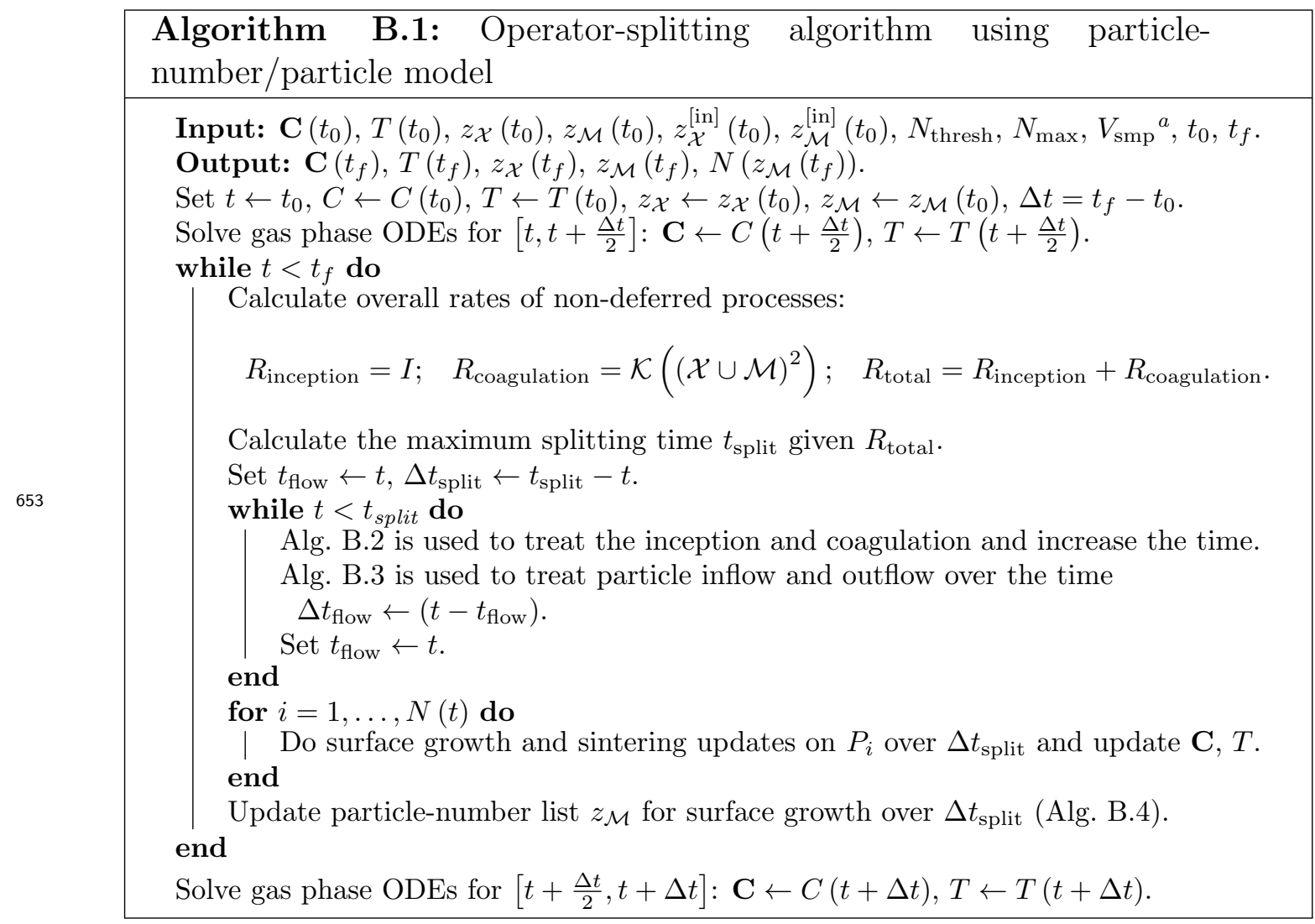

${ }^{a}$ Initially $V_{\mathrm{smp}}=N_{\max } / M_{0}^{\max }$ where $M_{0}^{\max }$ is an estimate of the maximum number density. 


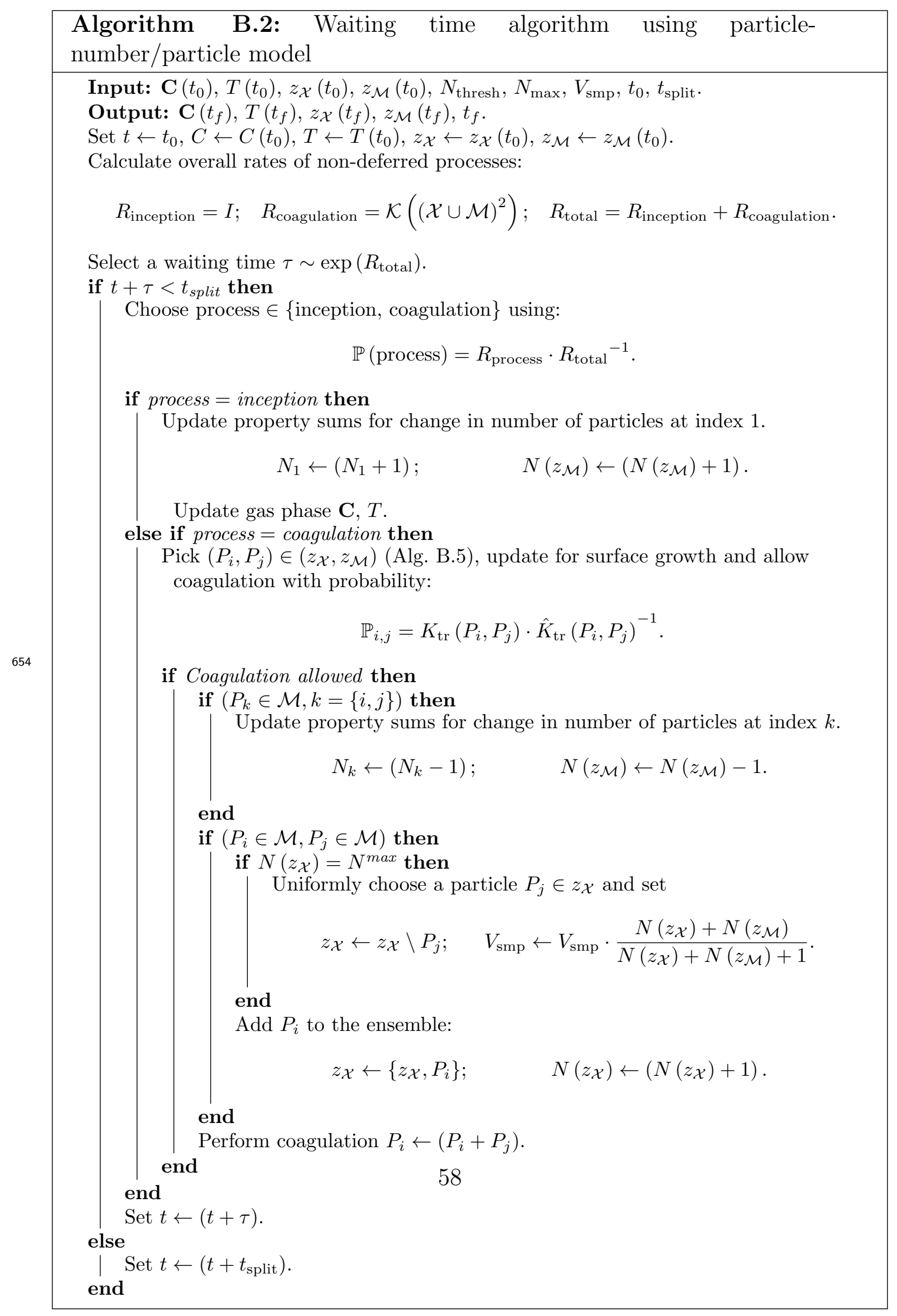




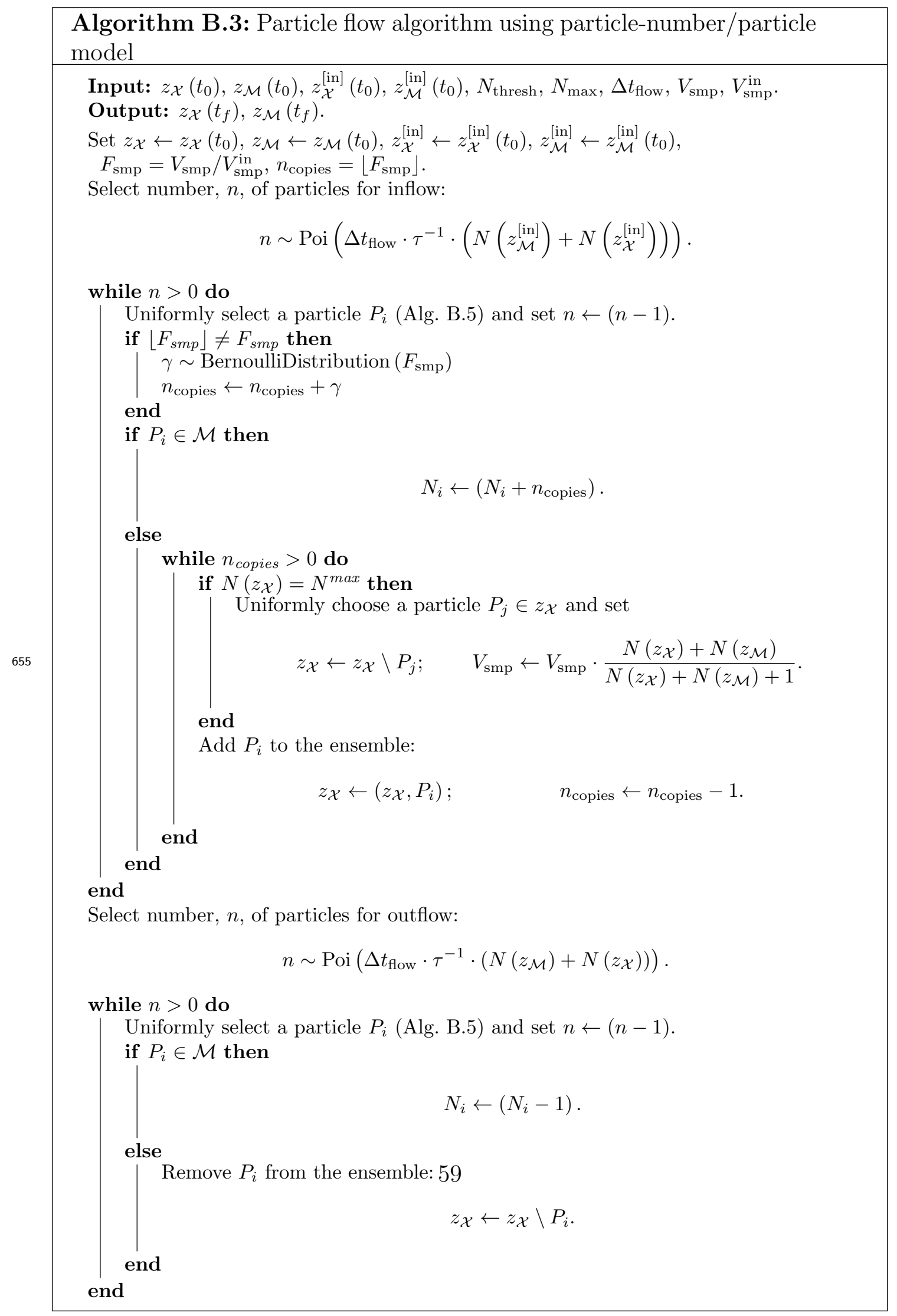




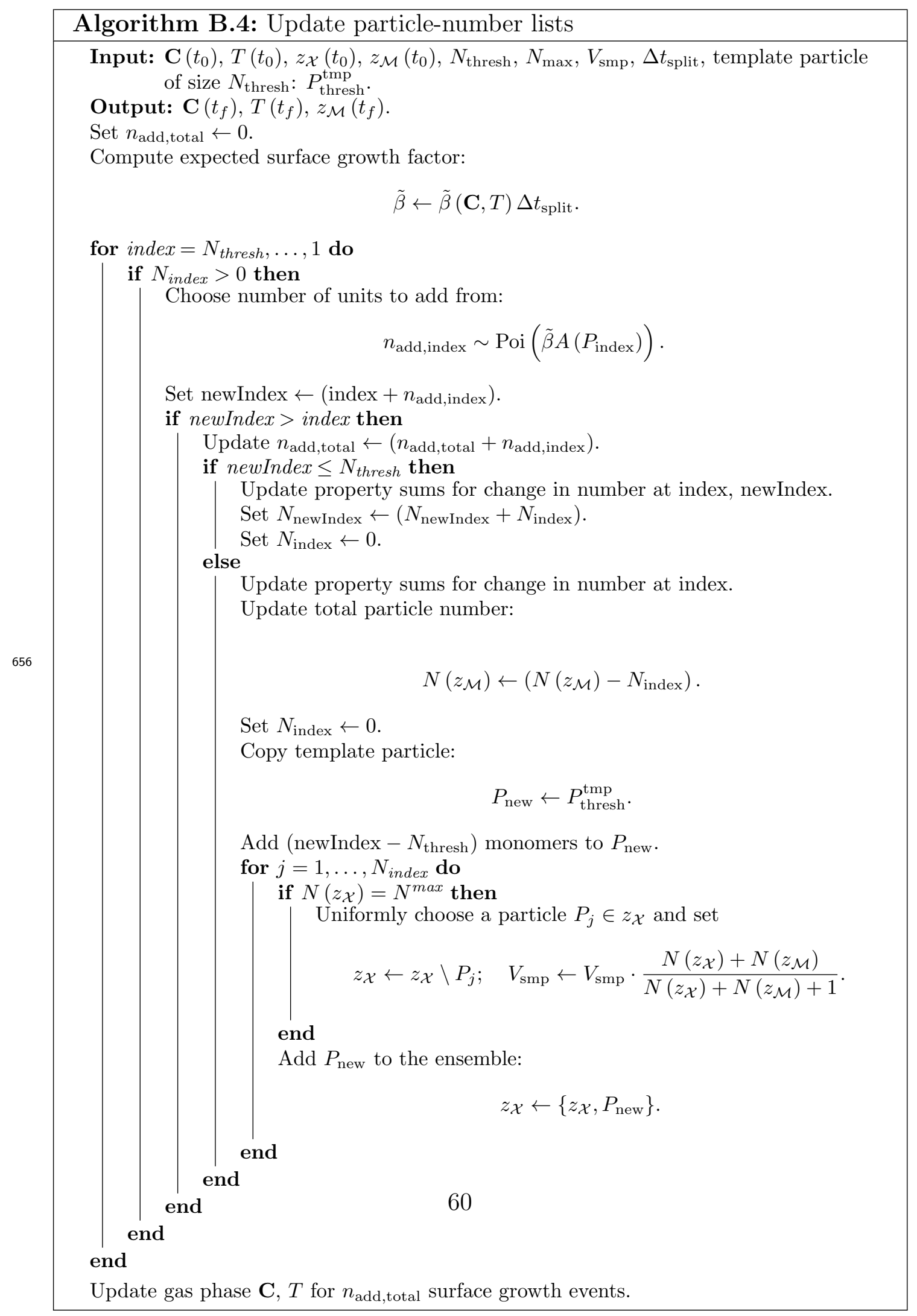




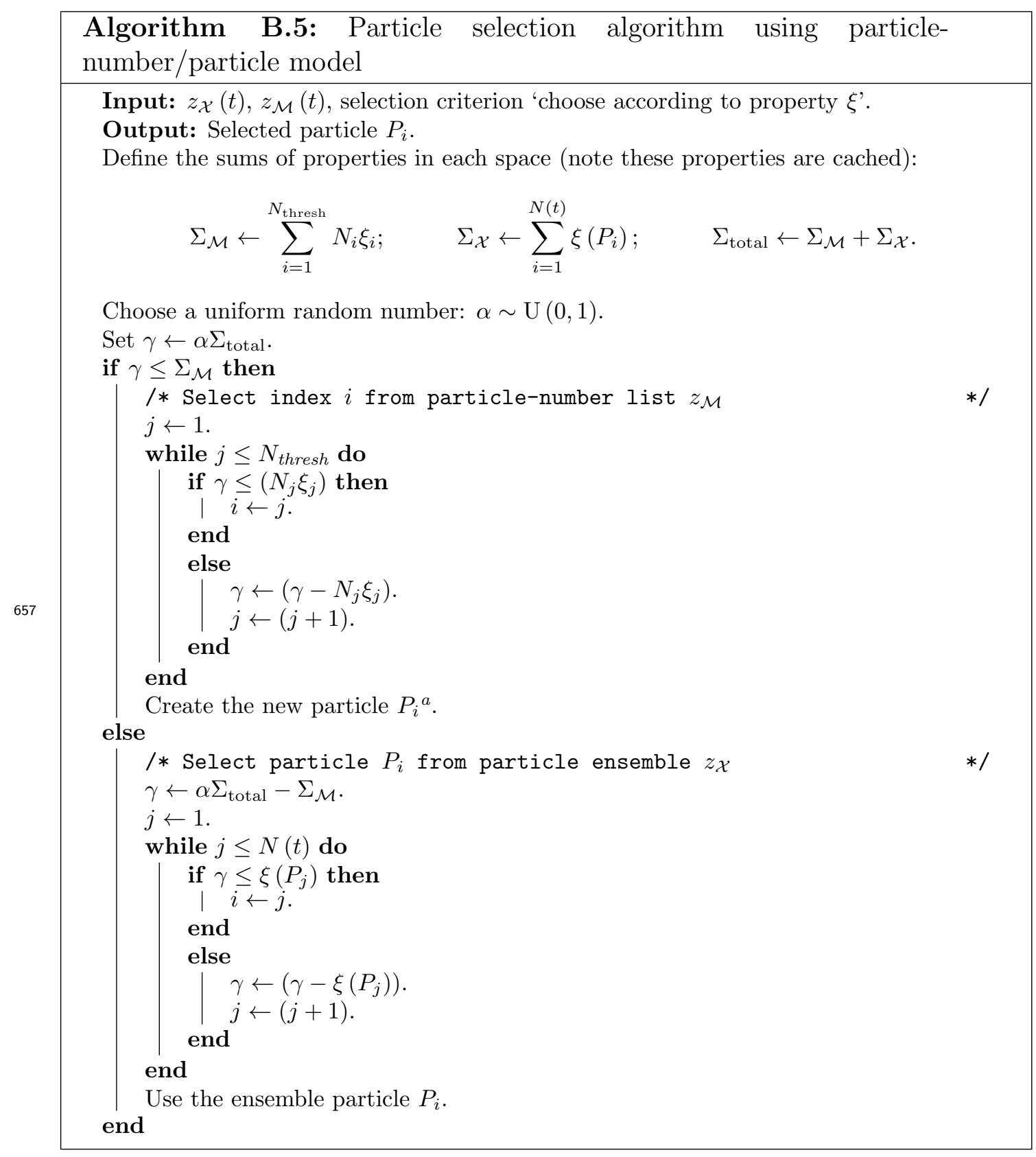

${ }^{a}$ Clone the particle with index $i$ from reference particle list 


\section{References}

[1] H. Wang, Formation of nascent soot and other condensed-phase materials in flames, Proceedings of the Combustion Institute 33 (2011) 41-67. doi:10.1016/j.proci.2010.09.009.

[2] H. K. Park, K. Y. Park, Control of Particle Morphology and Size in Vapor-Phase Synthesis of Titania, Silica and Alumina Nanoparticles, KONA Powder and Particle Journal 32 (2015) 85-101. doi:10.14356/kona.2015018.

[3] A. D. Randolph, M. A. Larson, Theory of particulate processes: analysis and techniques of continuous crystallization, 2nd ed., Academic Press, San Diego, 1988.

[4] R. DeVille, N. Riemer, M. West, Weighted Flow Algorithms (WFA) for stochastic particle coagulation, Journal of Computational Physics 230 (2011) 8427-8451. doi:10.1016/j.jcp.2011.07.027.

[5] E. Debry, B. Sportisse, B. Jourdain, A stochastic approach for the numerical simulation of the general dynamics equation for aerosols, Journal of Computational Physics 184 (2003) 649-669. doi:10.1016/S00219991(02)00041-4.

[6] N. Brilliantov, P. L. Krapivsky, A. Bodrova, F. Spahn, H. Hayakawa, V. Stadnichuk, J. Schmidt, Size distribution of particles in 
Saturn's rings from aggregation and fragmentation, Proceedings of the National Academy of Sciences 112 (2015) 9536-9541. doi:10.1073/pnas.1503957112.

[7] L. Malyshkin, J. Goodman, The Timescale of Runaway Stochastic Coagulation, Icarus 150 (2001) 314-322. doi:10.1006/icar.2001.6587.

[8] M. Kraft, Modelling of Particulate Processes, KONA Powder and Particle Journal 23 (2005) 18-35. doi:10.14356/kona.2005007.

[9] S. E. Pratsinis, P. T. Spicer, Competition between gas phase and surface oxidation of $\mathrm{TiCl}_{4}$ during synthesis of $\mathrm{TiO}_{2}$ particles, Chemical Engineering Science 53 (1998) 1861-1868. doi:10.1016/S0009-2509(98)000268.

[10] Y. Xiong, S. E. Pratsinis, Formation of agglomerate particles by coagulation and sintering - Part I. A two-dimensional solution of the population balance equation, Journal of Aerosol Science 24 (1993) 283-300. doi:10.1016/0021-8502(93)90003-R.

[11] C. Lindberg, J. Akroyd, M. Kraft, Developing breakage models relating morphological data to the milling behaviour of flame synthesised titania particles, Chemical Engineering Science 166 (2017). doi:10.1016/j.ces.2017.03.016.

[12] E. K. Yapp, D. Chen, J. Akroyd, S. Mosbach, M. Kraft, J. Camacho, H. Wang, Numerical simulation and parametric sensitivity study of particle size distributions in a burner-stabilised stag- 
nation flame, Combustion and Flame 162 (2015) 2569-2581. doi:10.1016/j.combustflame.2015.03.006.

[13] M. Sander, R. I. A. Patterson, A. Braumann, A. Raj, M. Kraft, Developing the PAH-PP soot particle model using process informatics and uncertainty propagation, Proceedings of the Combustion Institute 33 (2011) 675-683. doi:10.1016/j.proci.2010.06.156.

[14] M. S. Celnik, M. Sander, A. Raj, R. H. West, M. Kraft, Modelling soot formation in a premixed flame using an aromatic-site soot model and an improved oxidation rate, Proceedings of the Combustion Institute 32 (2009) 639-646. doi:10.1016/j.proci.2008.06.062.

[15] M. Sander, R. H. West, M. S. Celnik, M. Kraft, A Detailed Model for the Sintering of Polydispersed Nanoparticle Agglomerates, Aerosol Science and Technology 43 (2009) 978-989. doi:10.1080/02786820903092416.

[16] S. Shekar, W. J. Menz, A. J. Smith, M. Kraft, W. Wagner, On a multivariate population balance model to describe the structure and composition of silica nanoparticles, Computers \& Chemical Engineering 43 (2012) 130-147. doi:10.1016/j.compchemeng.2012.04.010.

[17] W. J. Menz, M. Kraft, A new model for silicon nanoparticle synthesis, Combustion and Flame 160 (2013) 947-958. doi:10.1016/j.combustflame.2013.01.014.

[18] R. H. West, M. S. Celnik, O. R. Inderwildi, M. Kraft, G. J. O. Beran, W. H. Green, Toward a Comprehensive Model of the Synthesis of $\mathrm{TiO}_{2}$ 
Particles from $\mathrm{TiCl}_{4}$, Industrial \& Engineering Chemistry Research 46 (2007) 6147-6156. doi:10.1021/ie0706414.

[19] A. Boje, J. Akroyd, S. Sutcliffe, J. Edwards, M. Kraft, Detailed population balance modelling of $\mathrm{TiO}_{2}$ synthesis in an industrial reactor, Chemical Engineering Science 164 (2017) 219-231. doi:10.1016/j.ces.2017.02.019.

[20] W. J. Menz, M. Kraft, The Suitability of Particle Models in Capturing Aggregate Structure and Polydispersity, Aerosol Science and Technology 47 (2013) 734-745. doi:10.1080/02786826.2013.788244.

[21] V. Stadnichuk, A. Bodrova, N. Brilliantov, Smoluchowski aggregationfragmentation equations: Fast numerical method to find steady-state solutions, International Journal of Modern Physics B 29 (2015) 1550208. doi:10.1142/S0217979215502082.

[22] A. J. Smith, C. G. Wells, M. Kraft, A new iterative scheme for solving the discrete Smoluchowski equation, Journal of Computational Physics 352 (2018) 373-387. doi:10.1016/j.jcp.2017.09.045.

[23] J. Koch, W. Hackbusch, K. Sundmacher, $\mathcal{H}$-matrix methods for linear and quasi-linear integral operators appearing in population balances, Computers \& Chemical Engineering 31 (2007) 745-759. doi:10.1016/j.compchemeng.2006.07.012.

[24] M. Frenklach, S. J. Harris, Aerosol dynamics modeling using the method of moments, Journal of Colloid and Interface Science 118 (1987) 252261. doi:10.1016/0021-9797(87)90454-1. 
[25] M. Frenklach, Method of moments with interpolative closure, Chemical Engineering Science 57 (2002) 2229-2239. doi:10.1016/S00092509(02)00113-6.

[26] J. Akroyd, A. J. Smith, R. Shirley, L. R. McGlashan, M. Kraft, A coupled CFD-population balance approach for nanoparticle synthesis in turbulent reacting flows, Chemical Engineering Science 66 (2011) 37923805. doi:10.1016/j.ces.2011.05.006.

[27] M. Y. Manuputty, J. Akroyd, S. Mosbach, M. Kraft, Modelling $\mathrm{TiO}_{2}$ formation in a stagnation flame using method of moments with interpolative closure, Combustion and Flame 178 (2017) 135-147. doi:10.1016/j.combustflame.2017.01.005.

[28] D. L. Marchisio, J. T. Pikturna, R. O. Fox, R. D. Vigil, A. A. Barresi, Quadrature method of moments for population-balance equations, AIChE Journal 49 (2003) 1266-1276. doi:10.1002/aic.690490517.

[29] R. McGraw, Description of aerosol dynamics by the quadrature method of moments, Aerosol Science and Technology 27 (1997) 255-265. doi:10.1080/02786829708965471.

[30] D. L. Marchisio, R. O. Fox, Solution of population balance equations using the direct quadrature method of moments, Journal of Aerosol Science 36 (2005) 43-73. doi:10.1016/j.jaerosci.2004.07.009.

[31] J. Akroyd, A. J. Smith, L. R. McGlashan, M. Kraft, Numerical investigation of DQMoM-IEM as a turbulent reaction closure, Chemical Engineering Science 65 (2010) 1915-1924. doi:10.1016/j.ces.2009.11.010. 
[32] S. Wu, E. K. Yapp, J. Akroyd, S. Mosbach, R. Xu, W. Yang, M. Kraft, A moment projection method for population balance dynamics with a shrinkage term, Journal of Computational Physics 330 (2017) 960-980. doi:10.1016/j.jcp.2016.10.030.

[33] M. J. Hounslow, R. L. Ryall, V. R. Marshall, A discretized population balance for nucleation, growth, and aggregation, AIChE Journal 34 (1988) 1821-1832. doi:10.1002/aic.690341108.

[34] S. Kumar, D. Ramkrishna, On the solution of population balance equations by discretization-I. A fixed pivot technique, Chemical Engineering Science 51 (1996) 1311-1332. doi:10.1016/0009-2509(96)88489-2.

[35] S. Kumar, D. Ramkrishna, On the solution of population balance equations by discretization-II. A moving pivot technique, Chemical Engineering Science 51 (1996) 1333-1342. doi:10.1016/0009-2509(95)00355$\mathrm{X}$.

[36] S. Kumar, D. Ramkrishna, On the solution of population balance equations by discretization-III. Nucleation, growth and aggregation of particles, Chemical Engineering Science 52 (1997) 4659-4679. doi:10.1016/S0009-2509(97)00307-2.

[37] S. Tsantilis, S. E. Pratsinis, Evolution of primary and aggregate particlesize distributions by coagulation and sintering, AIChE Journal 46 (2000) 407-415. doi:10.1002/aic.690460218.

[38] S. Matveev, A. Smirnov, E. Tyrtyshnikov, A fast numerical method 
for the Cauchy problem for the Smoluchowski equation, Journal of Computational Physics 282 (2015) 23-32. doi:10.1016/j.jcp.2014.11.003.

[39] J.-P. Bourgade, F. Filbet, Convergence of a finite volume scheme for coagulation-fragmentation equations, Mathematics of Computation 77 (2007) 851-883. doi:10.1090/S0025-5718-07-02054-6.

[40] M. Wulkow, A. Gerstlauer, U. Nieken, Modeling and simulation of crystallization processes using parsival, Chemical Engineering Science 56 (2001) 2575-2588. doi:10.1016/S0009-2509(00)00432-2.

[41] S. A. Matveev, D. A. Zheltkov, E. E. Tyrtyshnikov, A. P. Smirnov, Tensor train versus Monte Carlo for the multicomponent Smoluchowski coagulation equation, Journal of Computational Physics 316 (2016) 164-179. doi:10.1016/j.jcp.2016.04.025.

[42] W. J. Menz, J. Akroyd, M. Kraft, Stochastic solution of population balance equations for reactor networks, Journal of Computational Physics 256 (2014) 615-629. doi:10.1016/j.jcp.2013.09.021.

[43] A. Eibeck, W. Wagner, Stochastic Particle Approximations for Smoluchoski's Coagulation Equation, The Annals of Applied Probability 11 (2001) 1137-1165. doi:10.1214/aoap/1015345398.

[44] R. I. A. Patterson, Convergence of Stochastic Particle Systems Undergoing Advection and Coagulation, Stochastic Analysis and Applications 31 (2013) 800-829. doi:10.1080/07362994.2013.817245.

[45] C. G. Wells, A stochastic approximation scheme and convergence theorem for particle interactions with perfectly reflecting boundary con- 
ditions, Monte Carlo Methods and Applications 12 (2006) 291-342. doi:10.1515/156939606778705182.

[46] A. Maisels, F. Einar Kruis, H. Fissan, Direct simulation Monte Carlo for simultaneous nucleation, coagulation, and surface growth in dispersed systems, Chemical Engineering Science 59 (2004) 2231-2239. doi:10.1016/j.ces.2004.02.015.

[47] R. I. A. Patterson, W. Wagner, M. Kraft, Stochastic weighted particle methods for population balance equations, Journal of Computational Physics 230 (2011) 7456-7472. doi:10.1016/j.jcp.2011.06.011.

[48] G. Kotalczyk, F. Kruis, A Monte Carlo method for the simulation of coagulation and nucleation based on weighted particles and the concepts of stochastic resolution and merging, Journal of Computational Physics 340 (2017) 276-296. doi:10.1016/j.jcp.2017.03.041.

[49] K. F. Lee, R. I. A. Patterson, W. Wagner, M. Kraft, Stochastic weighted particle methods for population balance equations with coagulation, fragmentation and spatial inhomogeneity, Journal of Computational Physics 303 (2015) 1-18. doi:10.1016/j.jcp.2015.09.031.

[50] A. Eibeck, W. Wagner, Stochastic interacting particle systems and nonlinear kinetic equations, The Annals of Applied Probability 13 (2003) 845-889. doi:10.1214/aoap/1060202829.

[51] R. I. A. Patterson, J. Singh, M. Balthasar, M. Kraft, J. R. Norris, The Linear Process Deferment Algorithm: A new technique for solving 
population balance equations, SIAM Journal on Scientific Computing 28 (2006) 303-320. doi:10.1137/040618953.

[52] H. Babovsky, A hybrid numerical scheme for aerosol dynamics, in: Numerical Mathematics and Advanced Applications, Springer, 2008, pp. 425-432. doi:0.1007/978-3-540-69777-0.

[53] C. S. Lindberg, M. Y. Manuputty, E. K. Y. Yapp, J. Akroyd, R. Xu, M. Kraft, A detailed particle model for polydisperse titanium dioxide aggregates, Manuscript submitted for publication (2018).

[54] M. S. Celnik, R. I. A. Patterson, M. Kraft, W. Wagner, Coupling a stochastic soot population balance to gas-phase chemistry using operator splitting, Combustion and Flame 148 (2007) 158-176. doi:10.1016/j.combustflame.2006.10.007.

[55] S. Shekar, A. J. Smith, W. J. Menz, M. Sander, M. Kraft, A multidimensional population balance model to describe the aerosol synthesis of silica nanoparticles, Journal of Aerosol Science 44 (2012) 83-98. doi:10.1016/j.jaerosci.2011.09.004.

[56] R. H. West, G. J. O. Beran, W. H. Green, M. Kraft, First-Principles Thermochemistry for the Production of $\mathrm{TiO}_{2}$ from $\mathrm{TiCl}_{4}$, The Journal of Physical Chemistry A 111 (2007) 3560-3565. doi:10.1021/jp0661950.

[57] R. H. West, R. A. Shirley, M. Kraft, C. F. Goldsmith, W. H. Green, A detailed kinetic model for combustion synthesis of titania from $\mathrm{TiCl}_{4}$, Combustion and Flame 156 (2009) 1764-1770. doi:10.1016/j.combustflame.2009.04.011. 
${ }_{859}^{658]}$ W. J. Menz, R. I. A. Patterson, W. Wagner, M. Kraft, Application of stochastic weighted algorithms to a multidimensional silica par-

861 ticle model, Journal of Computational Physics 248 (2013) 221-234. doi:10.1016/j.jcp.2013.04.010. 